\title{
Wettability and Lenormand's Diagram
}

\author{
Bauyrzhan K. Primkulov ${ }^{1}$, Amir A. Pahlavan ${ }^{2}$, Xiaojing Fu ${ }^{3}$, \\ Benzhong Zhao ${ }^{4}$, Christopher W. MacMinn ${ }^{5}$, and Ruben Juanes ${ }^{1} \dagger$ \\ ${ }^{1}$ Massachusetts Institute of Technology, 77 Massachusetts Avenue, Cambridge, MA, USA \\ ${ }^{2}$ Princeton University, Olden St., Princeton, NJ, USA \\ ${ }^{3}$ University of California at Berkeley, McCone Hall, Berkeley, CA, USA \\ ${ }^{4}$ McMaster University, 1280 Main Street West, Hamilton, Canada \\ ${ }^{5}$ University of Oxford, Parks Road, Oxford, United Kingdom
}

(Received xx; revised xx; accepted xx)

Fluid-fluid displacement in porous media has been viewed through the lens of Lenormand's phase diagram since the late 1980s. This diagram suggests that the character of the flow is controlled by two dimensionless parameters: the capillary number and the viscosity ratio. It is by now well known, however, that the wettability of the system plays a key role in determining the pore-scale displacement mechanisms and macroscopic invasion patterns. Here, we endow Lenormand's diagram with the impact of wettability using dynamic and quasi-static pore-network models. By using the fractal dimension and the ratio of characteristic viscous and capillary pressures we delineate the five principal displacement regimes within the extended phase diagram: stable displacement, viscous fingering, invasion percolation, cooperative pore filling, and corner flow. We discuss the results in the context of pattern formation, displacement-front dynamics, pore-scale disorder, and displacement efficiency.

\section{Key words:}

\section{Introduction}

Patterns form during fluid-fluid displacement in porous media in many natural and industrial processes. As sand castles dry, air percolates into the sand matrix and the integrity of the structure depends strongly on the resulting moisture distribution (Richefeu et al. 2006; Møller \& Bonn 2007). In sugar processing, liquor-saturated charcoal packs are periodically cleansed with water, where channeling of the water phase is undesirable (Hill 1952). In refractory ceramics manufacturing, the ceramic matrix is infiltrated by molten metal, where higher degree of infiltration leads to more resilient ceramics (Léger et al. 2015). In hydrocarbon recovery, oil is produced by displacing it with water and higher displacement efficiency is more economically desirable (Datta et al. 2014). Understanding morphology of the displacement front during such processes is of great value.

Lenormand et al. (1988) presented a phase diagram (Fig. 1) to characterize fluid-fluid displacement in a porous medium with two dimensionless parameters: the mobility ratio $M \equiv \mu_{i} / \mu_{d}$ and the capillary number $\mathrm{Ca} \equiv \mu_{i} u / \gamma$, where $u$ is the characteristic velocity, $\gamma$ is the interfacial tension, and $\mu_{i}$ and $\mu_{d}$ are the dynamic viscosities of the invading and defending fluids, respectively. For high $\mathrm{Ca}$, viscous forces dominate over capillary forces.

$\dagger$ Email address for correspondence: juanes@mit.edu 
(a)

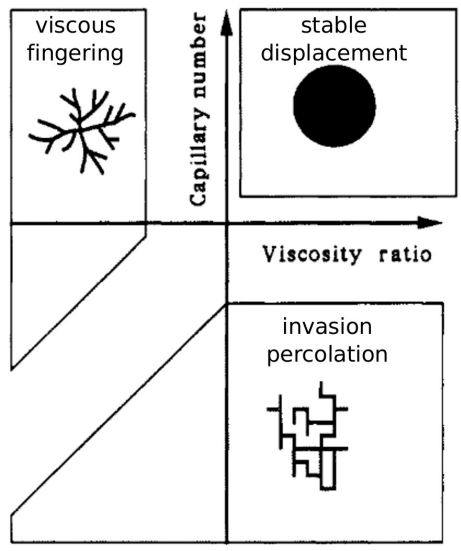

(b)

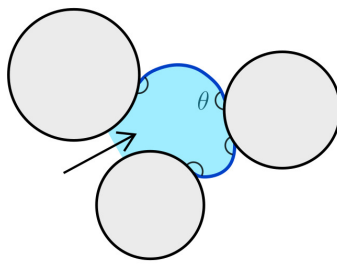

(c)

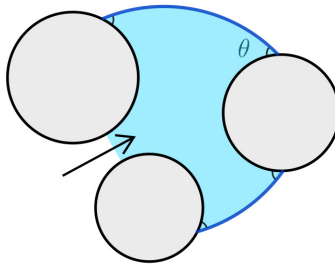

drainage

$\theta>90^{\circ}$

imbibition

$\theta<90^{\circ}$

For $M>1$ (favorable displacement) and high Ca, the displacement front is viscously stable and the invading fluid sweeps the porous medium compactly (Lenormand et al. 1988). For $M<1$ (unfavorable displacement) and high $\mathrm{Ca}$, the displacement front is subject to the Saffman-Taylor instability (1958) and develops a self-similar viscousfingering pattern (Hill 1952; Van Meurs 1957; Chuoke et al. 1959; Paterson 1984; Chen \& Wilkinson 1985; Måløy et al. 1985; Homsy 1987; Feder et al. 1989; Hinrichsen et al. 1989; Meakin et al. 1989; Ben Amar 1991a,b; Li et al. 2009; Patmonoaji et al. 2020). For low $\mathrm{Ca}$, capillary forces dominate over viscous forces and the displacement front advances via capillary invasion regardless of $M$ (Chandler et al. 1982; Wilkinson \& Willemsen 1983; Lenormand \& Zarcone 1985).

The wetting properties of the fluid-fluid-solid system are not a part of the original Lenormand et al. (1988) diagram, although significance of wettability has been acknowledged in Lenormand (1990). A number of studies have discussed the importance of wettability at both high and low Ca (Stokes et al. 1986; Cieplak \& Robbins 1988, 1990; Trojer et al. 2015; Holtzman \& Segre 2015; Zhao et al. 2016; Jung et al. 2016; Odier et al. 2017; Singh et al. 2017; Primkulov et al. 2018; Zhao et al. 2019; Primkulov et al. 2019). Wettability can be characterized by the contact angle $\theta$ at which the fluid-fluid interface meets the solid surface, measured from the invading fluid (Fig. 1b-c). For $\theta<90^{\circ}$, a more wetting fluid displaces a less wetting fluid and the process is called imbibition; for $\theta>90^{\circ}$, a less wetting fluid displaces a more wetting fluid and the process is called drainage. As the system transitions from strong drainage to weak imbibition, the displacement becomes more compact: for high $\mathrm{Ca}$ and $M<1$, the viscous fingers become wider (Stokes et al. 1986; Trojer et al. 2015; Zhao et al. 2016); for low Ca and all $M$, the displacement patterns are very compact (Cieplak \& Robbins 1988, 1990; Trojer et al. 2015; Zhao et al. 2016; Primkulov et al. 2018). When a capillary-dominated system (low Ca) is in strong imbibition, the displacement front advances by preferentially filling crevices and corners in the pore-space (corner-flow) (Levaché \& Bartolo 2014; Zhao et al. 2016; Odier et al. 2017; Primkulov et al. 2018).

The invading fluid does not always displace the defending fluid completely from invaded pores; corner-flow is one such case. Another instance of incomplete displacement takes 
place in strong drainage at high Ca (Park \& Homsy 1984; Zhao et al. 2016, 2019). Here, solid surfaces behind the displacement front remain coated with a film of defending fluid (Landau \& Levich 1988; Bretherton 1961; Zhao et al. 2016, 2019). The opposite happens in strong imbibition for high $\mathrm{Ca}$ and $M<1$ : films of invading fluid advance on the solid surfaces ahead of the bulk displacement front (Levaché \& Bartolo 2014; Zhao et al. 2016; Odier et al. 2017; Zhao et al. 2019).

Pore-network models are often used to simulate flow in porous media, as they are both intuitive and computationally inexpensive (Fatt 1956; Blunt \& Scher 1995; Celia et al. 1995; Øren et al. 1998; Constantinides \& Payatakes 2000; Patzek 2001; Blunt 2001; Joekar-Niasar \& Hassanizadeh 2012). The pore geometry in such models is approximated by a network of nodes and links, and the flow within each phase is assumed to be fully developed Poiseuille flow. The relatively low computational cost of such models makes them ideal for exploring full the $M-\mathrm{Ca}-\theta$ parameter space required for extending the original Lenormand diagram. No study to date, pore-network or otherwise, has produced a three-dimensional version of the Lenormand phase diagram, capturing gradual wettability-induced changes in the displacement patterns. The majority of porenetwork studies have targeted only a limited range of wettability conditions. While fluidfluid displacement has been extensively studied in separate sections of the $M$-Ca space in drainage (Chandler et al. 1982; Wilkinson \& Willemsen 1983; Chen \& Wilkinson 1985; Lenormand et al. 1988; Aker et al. 1998; Joekar-Niasar et al. 2010; Al-Gharbi \& Blunt 2005; Gjennestad et al. 2018), weak imbibition (Øren et al. 1998; Patzek 2001; Valvatne \& Blunt 2004), and strong imbibition with precursor wetting film flow through crevices and micro-roughness (Blunt \& Scher 1995; Vizika et al. 1994; Tzimas et al. 1997; Constantinides \& Payatakes 2000), only a few pore-network studies have explored the continuous transition in displacement patterns due to changes in $\theta$.

A substantial advance towards capturing continuous wettability-induced changes in displacement patterns was made by Cieplak \& Robbins $(1988,1990)$. Their model, which was designed for a 2D-porous medium comprised of a cylindrical obstacle array, reproduced experimentally observed compaction of the invading fluid as the system shifted from drainage to imbibition. This was done by introducing three pore-scale invasion mechanisms - burst, touch, and overlap (Fig. 3a-c) - whose relative frequencies shaped the displacement patterns at a given wettability. While this model was only valid for vanishing injection rates, Holtzman \& Segre (2015) extended it by including viscous effects for $M \ll 1$. The model allowed capturing the experimentally observed stabilization of fingering displacement patterns away from $\mathrm{Ca} \rightarrow 0$ (Trojer et al. 2015; Stokes et al. 1986).

At the same time, both pore-network models fell short of capturing three-dimensional effects that become important in strong imbibition. When $\theta<45^{\circ}$, the Laplace pressure of a wetting fluid in the corner between a post and a plate can be negative (Fig. 3d). Therefore, a strongly wetting invading fluid can advance predominantly through crevices between the top/bottom plates and the cylindrical obstacles. We account for this threedimensional mode of invasion by introducing a corner-flow event to the quasi-static model of Cieplak and Robbins (Primkulov et al. 2018). Specifically, we incorporate the corner flow event in the "moving-capacitor" framework (Primkulov et al. 2019), where we treat local fluid-fluid interfaces within a micromodel as analogs to capacitors in electrical circuits. Our approach in strong imbibition is similar to models by Blunt \& Scher (1995) and Constantinides \& Payatakes (2000), where displacement patterns are determined by competing flow through crevices and pore centers. However, unlike the model of Blunt \& Scher (1995), our model fully accounts for viscous pressure gradients and is therefore not limited to small length scales. Furthermore, our model does not 
pre-assign a distribution of micro-channels like the work of Constantinides \& Payatakes (2000); instead, connectivity of the invading fluid through crevices is determined by local micromodel geometry, and this connectivity evolves with the sequence of corner flow events. Ours is the first pore-network model to capture the continuous change in displacement patterns across all wettability conditions at arbitrary $\mathrm{Ca}$ and $M$. This feature, along with its computational efficiency, allows conducting an extensive parameter sweep over the entirety of $M-\mathrm{Ca}-\theta$ space. We utilize this model to build the first picture of a three-dimensional version of Lenormand's diagram, including an axis that represents wettability.

Recent studies have made strides in this direction, but stopped short of producing the full 3D diagram. Holtzman \& Segre (2015) outlined the changes in displacement patterns within $\mathrm{Ca}-\theta$ space for $M \ll 1$ using a pore-network model, excluding the possibility of corner flow. Hu et al. (2018) subsequently used continuum simulations to explore boundaries between viscous-dominated and capillary-dominated regimes for $M \approx 26$. This study was complemented by Lan et al. (2020), who used a dynamic pore-network model to explore the interplay between wettability and $\mathrm{Ca}$ for $M \approx 3 \cdot 10^{-3}$ which, like the model of Holtzman \& Segre (2015), neglected corner flow and was therefore limited to $\theta>45^{\circ}$. The phase diagrams produced in these studies correspond to a set of partial $\mathrm{Ca}-\theta$ slices of the $M-\mathrm{Ca}-\theta$ diagram we present in our manuscript.

In $\S 2$, we present our "moving-capacitor" pore-network framework in detail (Primkulov et al. 2019), which has been extended to all $\theta$ by incorporating corner flow events. Our model is based on the analogy between flow in porous media and currents in electrical circuits (Fatt 1956), and it treats the local fluid-fluid interfaces as a combination of batteries and capacitors. The model builds on many existing ideas in the porous-media community (Aker et al. 1998; Holtzman \& Segre 2015; Cieplak \& Robbins 1988, 1990; Blunt \& Scher 1995; Constantinides \& Payatakes 2000; Primkulov et al. 2018, 2019) and combines them into a single framework that is able to handle $M$-Ca space over all wettability conditions $\left(0^{\circ}<\theta<180^{\circ}\right)$. The model is built for the quasi-two-dimensional, paradigmatic case of randomly placed cylindrical pillars between the flat plates of a Hele-Shaw cell. We use the model to explore the principal flow regimes of fluid-fluid displacement in porous media $(\S 3)$. We then discuss the crossover from capillary invasion to viscous fingering under unfavorable displacement $(M<1)$ through pore-scale event statistics, symmetry of the displacement front, and autocorrelation of the flow field $(\S 4)$. Finally, we synthesize the results of over 7000 dynamic simulations into an extension of Lenormand's phase diagram that accounts for arbitrary wettability in $\S 5$.

\section{Method}

The model presented below builds on the analogy originally suggested by Fatt (1956), who pointed to the similarities between flow of a single fluid through a porous medium and flow of electrical current through a network of resistors. In this analogy, Ohm's and Kirchhoff's laws of electricity are analogous to the Hagen-Poiseuille law and conservation of mass for incompressible fluids, respectively. Therefore, resolving the viscous pressure drop due to flow through a particular network of tubes is equivalent to resolving the potential drop through an electrical circuit with identical topology.

This picture can be extended to two-phase flow by recognizing the similarities between local fluid-fluid interfaces and electrical capacitors. Electrical capacitors are traditionally used to store electrical charge: current builds up opposing charges across the capacitor plates, resulting in a step-change in electrical potential across the capacitor. This potential difference builds with current until a maximum is reached, which may result 
in dielectric breakdown of the capacitor. Similarly, when one fluid displaces another within a porous medium, the curvature of fluid-fluid interfaces increase as they advance into narrow sections of the pore geometry (i.e., pore throats), corresponding to higher Laplace pressure across the interface. Overcoming the maximum Laplace pressure (i.e., the capillary entry pressure) results in rapid invasion of the pore space ahead. This invasion is analogous to dielectric breakdown; however, unlike capacitors, the fluid-fluid interface will subsequently find the nearest pore throat and start re-building the Laplace pressure (thus curvature). We therefore refer to the model presented here as a "movingcapacitor" model.

We use the paradigmatic case of cylindrical obstacles in a Hele-Shaw cell as a quasi-2D porous medium (Cieplak \& Robbins 1988, 1990; Holtzman \& Segre 2015; Zhao et al. 2016; Jung et al. 2016; Holtzman 2016; Primkulov et al. 2018, 2019; Borgman et al. 2019; Hu et al. 2019). In this case, there is also an out-of-plane contribution to the Laplace pressure that is analogous to a battery at the displacement front. This "battery" represents the overall affinity of the porous medium to the invading fluid. For a constant and uniform gap between the plates, we assume that this out-of-plane curvature is fixed by the value of the contact angle, and is positive in drainage and negative in imbibition. By doing so, we neglect the effect of dynamic contact angle (Hoffman 1975; Voinov 1977; Cox 1986).

We organize the remainder of this discussion into three subsections. We begin by explaining how we construct the pore network in $\S 2.1$. Then, we discuss the single-phaseflow model in $\S 2.2$. Finally we present the details of the two-phase-flow model (i.e., the moving-capacitor model) in $\S 2.3$.

\subsection{Pore-Network Construction}

Unless otherwise specified, simulations are conducted in the geometry of a benchmark flow cell: a circular, patterned Hele-Shaw cell with a pore-throat size distribution that has a mean of $665 \mu \mathrm{m}$ and a standard deviation of $337 \mu \mathrm{m}$. The cell is $30 \mathrm{~cm}$ in diameter and has a centered injection port. We set the gap between the two plates of our flow cell to $100 \mu \mathrm{m}$. The benchmark flow geometry is constructed using MATLAB's pdemesh tool with meshing parameters tuned to match the pore-throat size distribution reported in Zhao et al. (2016). In this construction, posts are centered at the nodes of the triangular mesh, and their radii are set to $45 \%$ of the length of the shortest adjacent edge.

Each mesh triangle represents a pore [Fig. 2(a)], so we can build the pore-network incidence matrix (Strang 2007) by examining the adjacency of the triangles. We number all pores and adopt the convention that pore connections are oriented in the direction of increasing pore number. As such, the incidence matrix of the network presented in Fig. 2(a) is

$$
\boldsymbol{A}=\left[\begin{array}{cccc}
-1 & 1 & 0 & 0 \\
0 & -1 & 1 & 0 \\
-1 & 0 & 0 & 1
\end{array}\right]
$$

where rows and columns of $\boldsymbol{A}$ represent edges and nodes, respectively. Here, 1 and -1 indicate entering and leaving the node, respectively. For example, edge 1 in eq. (2.1) is directed from node 1 to node 2 .

We also make use of the diagonal conductance matrix $\boldsymbol{C}$, whose elements are the hydraulic conductivities of the network edges. The elements of $\boldsymbol{C}$ can be calculated as $c=\pi r^{4} / 8 \mu L$, assuming fully developed Hagen-Poiseuille flow through a rectangular tube with hydraulic radius $r$ and length $L$, which correspond to pore-throat radius and distance between pore centers in a micromodel geometry, respectively. 
6

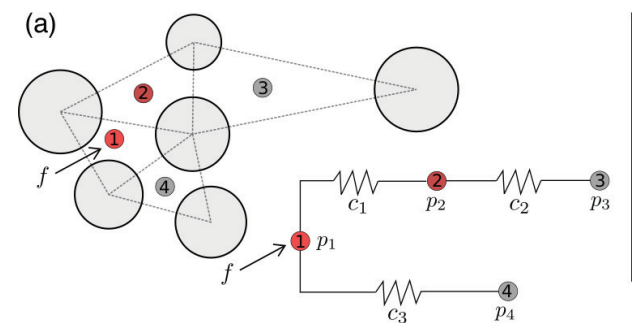

(c)
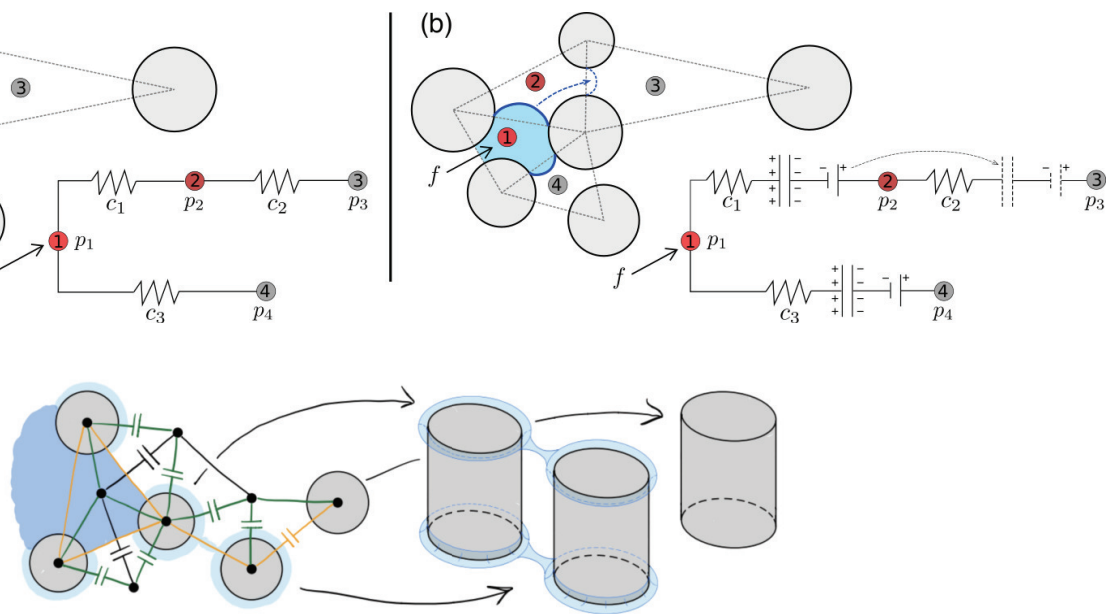

FIGURE 2. Schematic of flow through a porous medium and the analog electrical circuit for (a) single-phase flow and (b) two-phase flow. Nodes of the electrical circuit correspond to pore centres. Viscous pressure drop is analogous to potential drop through resistors, and fluid-fluid interfaces are analogous to a combination of a capacitor and a battery. (c) Schematic of the dynamic pore-network model in strong imbibition $\left(\theta<45^{\circ}\right)$, where capacitors are placed at the fluid-fluid interfaces. Nodes are placed at pore and post centers; black, orange, and green edges correspond to pore-to-pore, post-to-post, and pore-to-post edges respectively.

\subsection{Single-Phase Flow}

The difference in potential across the network edges can be obtained from the incidence matrix as $\boldsymbol{e}=-\boldsymbol{A} \boldsymbol{p}$ (Strang 2007). Here, $\boldsymbol{p}$ is an array of node potentials, which in the example of Fig. 2a would read as $\boldsymbol{p}=\left(p_{1}, p_{2}, p_{3}, p_{4}\right)^{T}$. The network currents can be calculated from the potential difference as $\boldsymbol{q}=\boldsymbol{C} \boldsymbol{e}$, where the example of Fig. 2a would have $\boldsymbol{q}=\left(q_{1}, q_{2}, q_{3}\right)^{T}$ and

$$
\boldsymbol{C}=\left[\begin{array}{ccc}
c_{1} & 0 & 0 \\
0 & c_{2} & 0 \\
0 & 0 & c_{3}
\end{array}\right]
$$

At the same time, currents must obey Kirchhoff's current law (or mass conservation in fluid flow), $\boldsymbol{A}^{T} \boldsymbol{q}=\boldsymbol{f}$, where $\boldsymbol{f}$ is the array of current sources at the nodes and would read $\boldsymbol{f}=(f, 0,0,0)^{T}$ for the example in Fig. 2a. After eliminating $\boldsymbol{e}$, single-phase flow through the network is captured by the following system of equations:

$$
\begin{aligned}
\boldsymbol{q} & =-\boldsymbol{C A} \boldsymbol{p}, \\
\boldsymbol{A}^{T} \boldsymbol{q} & =\boldsymbol{f} .
\end{aligned}
$$

Eliminating $\boldsymbol{q}$, the node potentials are given by

$$
\boldsymbol{p}=-\left(\boldsymbol{A}^{T} \boldsymbol{C} \boldsymbol{A}\right)^{-1} \boldsymbol{f} .
$$

We set constant-flow boundary conditions at the inlet pores (at the center of the flow cell) and zero-pressure boundary conditions at the outlet pores (at the edges of the flow cell).

\subsection{Two-Phase Flow: Moving Capacitors}

To extend the model to two-phase flow, we take advantage of the analogy between a capacitor and a fluid-fluid interface, where the drop in potential across the capacitor 
plates is analogous to the Laplace pressure. Consider the network diagram in Fig. 2(b). Initially, the capacitor is between nodes 1 and 2. As the current flows through the network, the capacitor accumulates charge and the potential difference across its plates builds. Capacitors with high accumulated potential difference hinder further flow, redirecting it elsewhere. Once the capacitor is filled to its maximum capacity, we allow it to advance to the next stable configuration at the neighboring edges (between nodes 2 and 3).

Our previous work on quasi-static fluid-fluid displacement (Primkulov et al. 2018) provides a framework for deciding how and when capacitors move. For any given configuration of the fluid-fluid interface (capacitor locations), the quasi-static model predicts both the critical Laplace pressures $\left(\Delta p_{\text {crit }}\right)$ and the type of interface instability. The type of instability event (i.e., burst, touch, overlap, or corner flow; see Fig. 3) determines the next stable interface configuration (Cieplak \& Robbins 1990, 1988; Primkulov et al. 2018). The critical Laplace pressure for burst, touch, and overlap events can be written as

$$
\Delta p_{\text {crit }}=\gamma\left(\frac{1}{r_{\text {in }}}+\frac{1}{r_{\text {out }}}\right),
$$

where $1 / r_{\text {out }}=2 \cos \theta / h$ is the out-of-plane curvature of the fluid-fluid interface and $1 / r_{\text {in }}$ is the in-plane curvature that corresponds to either burst, touch, or overlap configuration (Fig. 3a-c). Burst events correspond to the highest stable in-plane curvature of the interface between two posts (Fig. 3a). Touch events correspond to the interface coming in contact with a nearby post (Fig. 3b). Overlap events occur when two neighboring interfaces coalesce within the pore space (Fig. 3c). When $\theta<45^{\circ}$, the invading fluid tends to coat the corners between the posts and top/bottom plates. Corner-flow events occur when the horizontal extent of such meniscus reaches the nearest uncoated post (Fig. 3d). If these corner menisci instead overlap mid-post, they form a capillary bridge that expands spontaneously to the nearest post (Fig. 3e). The value of $\Delta p_{\text {crit }}$ for cornerflow and capillary bridge events is calculated from the total curvature of the meniscus configurations depicted in Fig. 3d-e. A more detailed description of all pore-scale events is given in Primkulov et al. (2018).

We assume that the pressure drop across a capacitor at time $t$ can be written as $\Delta p_{\text {crit }} \Phi(t)+\Delta p_{\min }(1-\Phi(t))$, where the filling ratio $\Phi(t)$ measures the fraction of the throat filled with invading fluid (Holtzman \& Segre 2015). A throat volume is defined as $2 r L h$. We chose $\Delta p_{\min }$ so that it is equal to the smallest value of $\Delta p_{\text {crit }}$ minus the standard deviation of $\Delta p_{\text {crit }}$ within the network. This choice ensures that all menisci have the same Laplace pressure when corresponding throats are empty. Taking into account the direction of the edges (an array $\boldsymbol{d}(t)$ consisting of 1 and -1 for edges directed towards and away from the defending fluid, respectively), the total pressure drop across the network edges can be written as $\boldsymbol{e}=\boldsymbol{b}-\boldsymbol{A} \boldsymbol{p}$, where non-zero components of pressure drop array $\boldsymbol{b}(t)$ are written as $-\boldsymbol{d}(t)\left[\Delta p_{\text {crit }} \Phi(t)+\Delta p_{\min }(1-\Phi(t))\right]$. Therefore, the equations governing two-phase flow through the network are

$$
\left[\begin{array}{cc}
\boldsymbol{C}^{-1}(t) & \boldsymbol{A} \\
\boldsymbol{A}^{T} & \mathbf{0}
\end{array}\right]\left[\begin{array}{l}
\boldsymbol{q}(t) \\
\boldsymbol{p}(t)
\end{array}\right]=\left[\begin{array}{c}
\boldsymbol{b}(t) \\
\boldsymbol{f}
\end{array}\right] .
$$

We now discuss time-stepping method in our two-phase flow model. After we initialize the interface locations within the "circuit", we use adaptive Forward Euler time-stepping to update the filling ratios of the network edges at the interface, $\Phi(t)$. We ensure that no pore throat is filled in a single time step (Aker et al. 1998). After every time step, we use the effective viscosity (Aker et al. 1998; Holtzman \& Segre 2015) $\mu=\mu_{i} \Phi(t)+\mu_{d}(1-\Phi(t)$ ) 
(a)

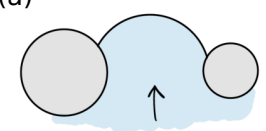

(b)

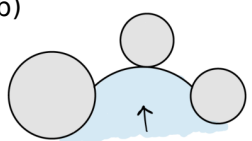

(c)

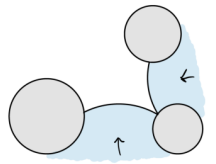

(d)

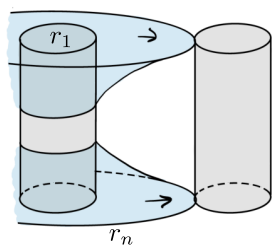

(e)

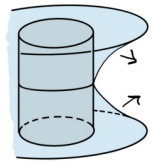

(f)
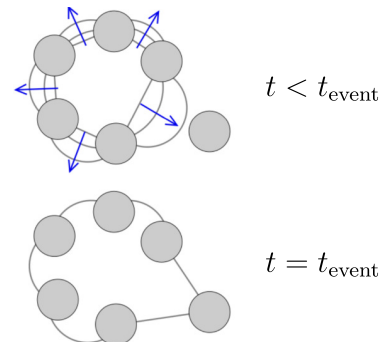

$t=t_{\text {event }}$

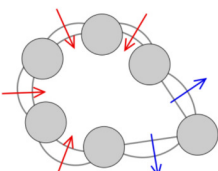

$t>t_{\text {event }}$

FiguRE 3. (a) A burst event occurs when the interface pushes past its highest stable curvature. (b) A touch event occurs when the interface touches the post ahead; (c) An overlap event occurs when two neighboring interfaces touch and coalesce, filling the pore cooperatively; (d) A corner-flow event occurs when a corner meniscus touches and coats the neighbouring post; (e) A capillary-bridge event occurs when corner menisci coalesce mid-post before reaching the next post; (f) A sequence of interface configurations before and after pore-invasion event at $t=t_{\text {event }}$ in capillary-dominated displacement. Figure adapted from Primkulov et al. (2018).

to update the conductivity matrix $\boldsymbol{C}(t)$ and resolve the flow via Eq. (2.6) with updated pressure drops across capacitors.

Whenever we encounter a time step $(\Delta t)$ where one of the components of $\Phi(t)$ is greater than 1 we repeat the time step with an adjusted $\Delta t$ until the unstable edge is exactly filled. Then, we remove the filled capacitor and replace it with empty capacitors at locations based on the type of instability that the quasi-static model outputs for the corresponding network edge (Primkulov et al. 2018). Newly added capacitors are initialized with $\Phi=0$ and accumulate potential drop as the above steps are repeated.

The typical solution of equation (2.6) in capillary-dominated regime produces the invasion sequence depicted in Fig. 3f, which can be separated into three steps: (i) interface curvatures build slowly across the displacement front $\left(t<t_{\text {event }}\right)$; (ii) one of the interfaces reaches a "burst", "touch", or "overlap" configuration, and the corresponding pore is instantaneously invaded with new interfaces having zero in-plane curvature and $\Phi$ $\left(t=t_{\text {event }}\right)$; (iii) the invading fluid redistributes to equalize the Laplace pressures at the displacement front $\left(t>t_{\text {event }}\right)$. The displacement front spends the majority of its time in step (i). Since capturing the short-time dynamics of invasion events (e.g., Haines 1930) was not the primary objective of this work, we chose to make step (ii) instantaneous, and chose a relatively coarse $\Delta t$, with (iii) taking up only a few time steps between pore-invasion events. As a result, having $\Phi=0$ correspond to zero in-plane curvature (our model) and having $\Phi=0$ correspond to a negative in-plane curvature (expected experimentally) would only make an appreciable difference in the short-time single-pore dynamics, which is outside the scope of interest of this study. Indeed, it is likely that a fully resolved model of the interface at the pore level is needed to capture these shorttimescale dynamics.

While our model of two-phase flow allows for re-emptying of network edges at the interface (Fig. 3f), our current implementation prohibits instability events in the reverse direction for simplicity of bookkeeping. 


\subsection{Moving-capacitor Model in Strong Imbibition}

When $\theta<45^{\circ}$, a total curvatures of corner a meniscus (Fig. 3d,e) can be negative. This means that at some $\theta<45^{\circ}$, invading fluid may advance by coating post corners instead of filling pore volumes. This was demonstrated in strong imbibition experiments of Zhao et al. (2016). Our treatment of strong imbibition fits naturally into the two-phase model described above, where the lowest $\Delta p_{\text {crit }}$ corresponds to either corner-flow (Fig. 3d) or capillary bridge event (Fig. 3e). Below, we highlight a few distinguishing features of the "moving-capacitor" model for $\theta<45^{\circ}$.

The overall flow network accounts for three distinct components: (i) a pore network, where nodes are pore centers and edges are pore-to-pore channels (black network in Fig. 2c), (ii) a post corner network, where nodes are placed at the centers of posts and edges are post-to-post connections (orange network in Fig. 2c), and (iii) a network connecting post centers to pore centers (green network in Fig. 2c).

Hydraulic radii of post-to-post and pore-to-post connections are taken as twice the ratio of channel cross sectional area to its wetted perimeter, which are calculated from the shape of the corner meniscus at its critical Laplace pressure (Fig. 3d,e). Volume assigned to a corner meniscus is defined to be $2 \pi r_{\text {post }} \pi r_{\text {hydr }}^{2}$, where $r_{\text {post }}$ and $r_{\text {hydr }}$ are radius of the post and hydraulic radius of the meniscus respectively.

In post-to-post and pore-to-post capacitors, the value of $\Phi$ is assigned to a post, so that capacitors belonging to the same post have identical Laplace pressures at any given time. When a new post is coated, only one capacitor is removed from the network, the capacitor at the post-to-post connection (Fig. 2c), and new capacitors are added at the fluid-fluid boundaries of the new post.

Another distinction between the model we present here from the original "movingcapacitor" model is that the corner events depicted in Fig. 3d,e can trigger pore invasion. The volume of each pore in our network is bounded by three posts. Therefore, if all three posts experience corner events, the oil phase within the pore space pinches off and pore gets filled with invading fluid (Odier et al. 2017).

Finally, our model assumes perfectly smooth surfaces and leaves out the role that surface roughness, dynamic contact angle, and potential precursor films may play in the fluid-fluid displacement experiments. While our model on this idealized substrate predicts no corner flow when $h=100 \mu \mathrm{m}$ (Primkulov et al. 2018), experiments detect the onset of corner flow for $\theta$ somewhere between $7^{\circ}$ and $60^{\circ}$ (Zhao et al. 2016). This discrepancy between experiment and the model is reconciled through a fitting parameter that we discuss in detail in Appendix A.

\section{Principal Flow Regimes}

We begin our discussion by exploring the five principal regimes of fluid-fluid displacement in porous media: (i) viscous fingering, (ii) stable displacement, (iii) invasion percolation, (iv) cooperative pore filling, and (v) corner flow. We anchor our discussion of principal flow regimes around a few key metrics that help to characterize and distinguish the regimes:

- Fractal dimension $D_{f}$ is a measure of how a pattern fills the space in which it is embedded. For a two-dimensional pattern, $D_{f}$ varies between 1 (for a line) and 2 (for a compact object). We calculate $D_{f}$ with the box-counting method (Kenkel \& Walker 1996). Following this method, we tile our flow patterns with boxes of size $\epsilon$ and count the number of boxes $N$ of that size needed to cover the pattern. We repeat this process 
for a sequence of $\epsilon$ and take $D_{f}$ to be the slope of $N$ against $\epsilon$ on a log-log plot (see Primkulov et al. (2018) for more details).

- Finger width $w / a$ is the ratio of mean finger width to mean pore size. We estimate $w / a$ following a scheme detailed in Primkulov et al. (2018), which is an adaptation of an approach by Cieplak \& Robbins $(1988,1990)$. Briefly, we divide our images into slices and record the mean size $w$ of one-dimensional clusters containing the pattern. We repeat the same process for an image where we treat the entire pore space as a pattern and record the mean pore throat size as $a$.

- Modified capillary number $\left(\mathrm{Ca}^{*}\right)$ measures the fraction of characteristic viscous to capillary pressures in our setup. We take

$$
\mathrm{Ca}^{*}=\frac{\Delta p_{\text {visc }}}{\Delta p_{\text {cap }}}=\frac{\max (\mathrm{Ca}, \mathrm{Ca} / M)}{\left|\Delta p_{\text {crit }}\right|} \frac{\gamma R}{a h},
$$

after expanding the characteristic pressure drop as $\Delta p_{\text {visc }}=\max \left(\mu_{i}, \mu_{d}\right) u R / a h$, where $R$ is the radius of the Hele-Shaw cell. The term $\max (\mathrm{Ca}, \mathrm{Ca} / M)$ ensures that the greater viscous forces are taken into account, and the magnitude of critical Laplace pressure $\left|\Delta p_{\text {crit }}\right|$ is taken directly from simulations.

All of these metrics are time-dependent. We evaluate $D_{f}$ and $w / a$ at the moment of breakthrough, when the invading fluid first reaches the outer boundary of the flow cell. The characteristic velocity $u$ used in calculating $\mathrm{Ca}$ and $\mathrm{Ca}^{*}$ is taken as $Q / 2 \pi r_{\min }$, where $r_{\min }$ is the radial distance of the post closest to center of the Hele-Shaw cell. Additionally, we define a directional flow rate as the mean flow rate along different directions of the radial flow cell. We do so by dividing the flow cell into $10^{\circ}$ sectors and calculating the mean flow rate for each sector as time progresses.

\subsection{Stable Displacement $\left(D_{f}=1.93, w / a=37, C a^{*}>1\right)$}

When a more viscous fluid displaces a less viscous fluid $(M>1)$, the displacement front is hydrodynamically stable (Saffman \& Taylor 1958) because viscous forces smooth perturbations.

Simulations at $M=10^{3}, \mathrm{Ca}=10^{-1}, \theta \in\left[46^{\circ}, 180^{\circ}\right]$ produce nearly perfectly circular patterns (Fig. 4). The injection pressure increases as the displacement progresses (Fig. 4a), with most of the pressure drop taking place in the invading fluid (Fig. 4d). The flow rate is radially symmetric, decreasing with radius (Fig. 4b,e), and pattern symmetry is maintained throughout (Fig. 4c).

\subsection{Viscous Fingering $\left(D_{f}=1.63, w / a=2.1, C a^{*}>1\right)$}

Stable displacement is often desirable, but not always attainable in industrial applications like oil recovery (Chuoke et al. 1959) and sugar processing (Hill 1952). Viscous fingers develop under potential flow when a less-viscous fluid displaces a more viscous one $(M<1)$.

In Fig. 5, we highlight the signatures of viscous fingering for the benchmark pore geometry. The simulation in Fig. 5 is conducted for $\mathrm{Ca}=10^{-1}, M=10^{-3}, \theta=170^{\circ}$. As the displacement advances, the injection pressure decreases (Fig. 5a) because the majority of the pressure drop takes place in the defending fluid (Fig. 5d). Although the pressure appears to decrease smoothly in time, removing the global trend from the signal would expose fluctuations due to intermittent activity at the displacement front (Primkulov et al. 2019). As the fingers develop and grow, they focus the flow along their main branches (Fig. 5b,e). The displacement pattern remains radially symmetric throughout (Fig. 5c). In fact, the diffusive signature of the pressure field in the defending fluid is what 

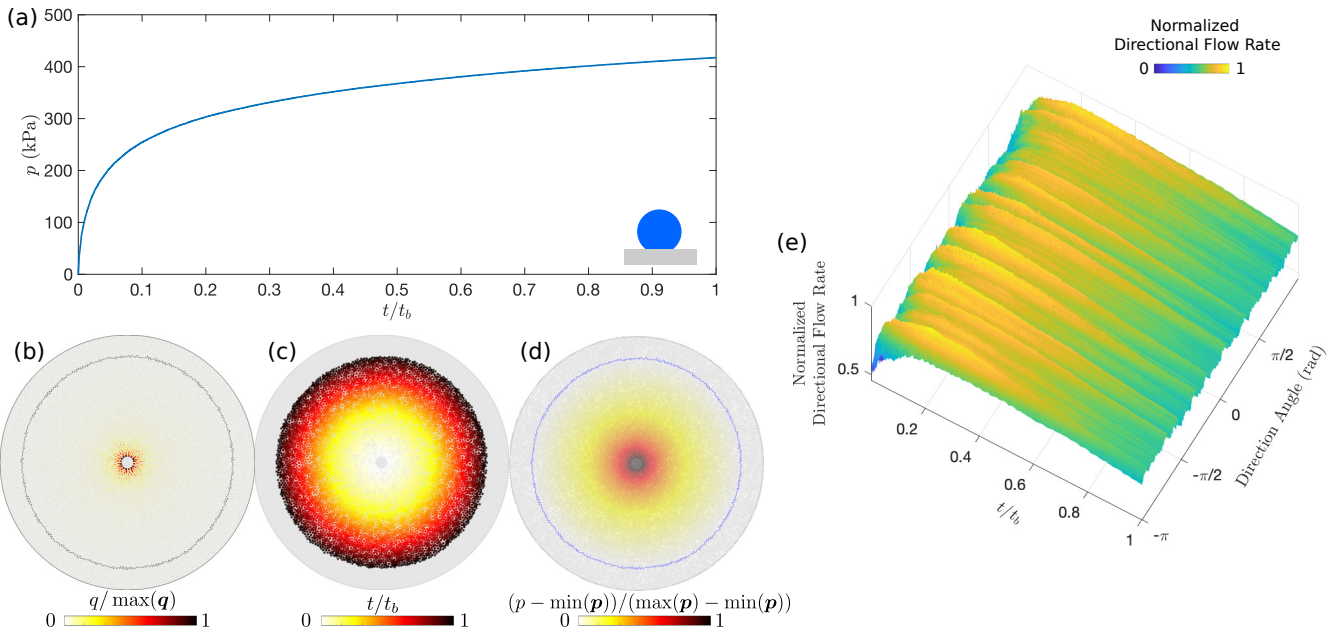

Figure 4. Stable displacement in the benchmark geometry for $\mathrm{Ca}=10^{-1}, M=10^{3}$, and $\theta=170^{\circ}$; (a) the injection pressure increases monotonically ( $t_{b}$ is the breakthrough time); (b) flow rates within the network show radial symmetry and radially-decreasing intensity $(\max (\boldsymbol{q})$ is the largest local flowrate at given $t)$; (c) pore-invasion times reflect the radial symmetry in pattern growth; (d) pore-pressure distribution, where pressure gradients are significant only in the invading fluid $(\max (\boldsymbol{p})$ is the largest local pressure at given $t)$; (e) the evolution of the directional flow rate is indicative of continuous compact flow, where apparent ridges are artifacts due to discrete pore throats with high flow rates near the cell center.
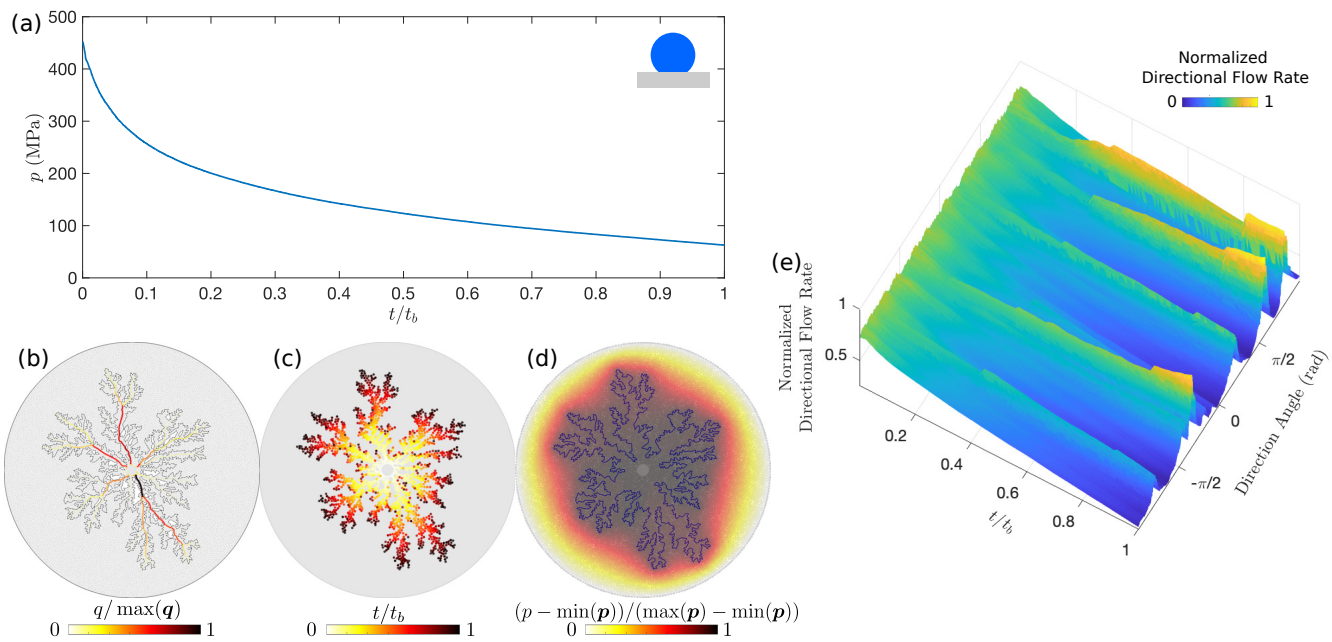

Figure 5. Viscous fingering in the benchmark geometry for $\mathrm{Ca}=10^{-1}, M=10^{-3}$, and $\theta=170^{\circ}$; (a) the injection pressure decreases monotonically in time $\left(t_{b}\right.$ is the breakthrough time); (b) flowrates within the network are pronounced along the main branches of the viscous fingers $(\max (\boldsymbol{q})$ is the largest local flowrate at given $t)$; (c) pore-invasion times reflect the radial symmetry in pattern growth; (d) pore-pressure distribution, where most pressure changes occur within the defending fluid $(\max (\boldsymbol{p})$ is the largest local pressure at given $t)$; (e) the evolution of the directional flow rate shows persistent (rather than sporadic) growth of viscous fingers.

generates the striking similarity between viscous fingering and other patterns in nature, such as diffusion-limited aggregation (DLA) (Meakin et al. 1989), dielectric breakdown of materials (Niemeyer et al. 1984), and spreading of fire fronts (Conti \& Marconi 2010). The diffusive pressure field arises from Darcy flow and incompressibility, which lead to 

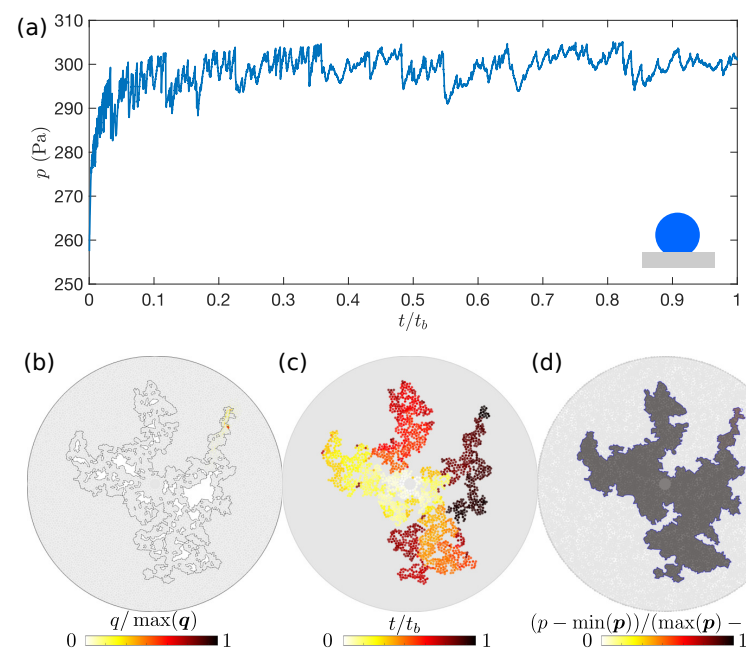

(d)

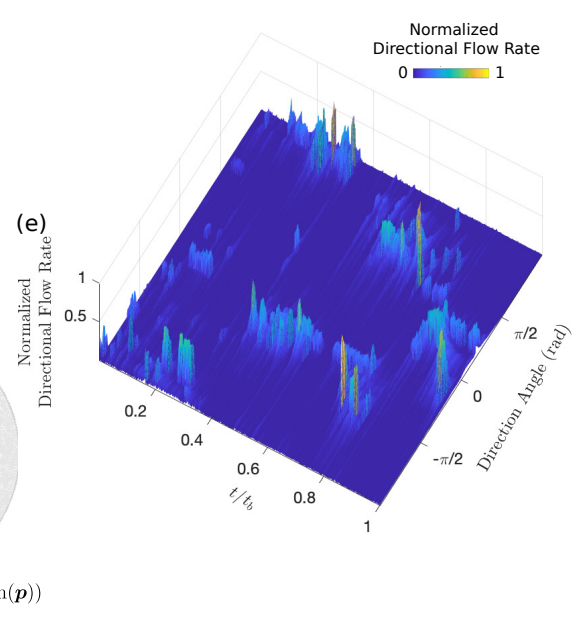

Figure 6. Invasion percolation in the benchmark geometry for $\mathrm{Ca}=10^{-7}, M=1$, and $\theta=170^{\circ}$; (a) the injection pressure fluctuates sharply due to pore-invasion events $\left(t_{b}\right.$ is the breakthrough time); (b) flowrates within the network are very localized, only a small fraction of the pore space is hydrodynamically active at any given time $(\max (\boldsymbol{q})$ is the largest local flowrate at given $t$ ); (c) pore-invasion times show asymmetric pore invasion clusters; (d) the pore-pressure distribution is uniform within each fluid $(\max (\boldsymbol{p})$ is the largest local pressure at given $t)$; (e) the evolution of the directional flow rate shows intermittency in flow direction.

$\nabla^{2} p=0$ in the defending fluid, which is identical to the diffusive solute concentration field in DLA (Paterson 1984).

\subsection{Invasion Percolation $\left(D_{f}=1.8, w / a=3, C a^{*}<1\right)$}

When the invading fluid advances very slowly and viscous forces are negligible $(\mathrm{Ca} \rightarrow$ $0)$, the flow is governed exclusively by capillary forces. In drainage $\left(\theta>90^{\circ}\right)$, the invading fluid advances mainly through burst events and the flow is well captured by the invasionpercolation model (Chandler et al. 1982; Wilkinson \& Willemsen 1983; Lenormand \& Zarcone 1985).

We explore the characteristics of invasion percolation by simulating fluid-fluid displacement at $\mathrm{Ca}=10^{-7}, M=1$, and $\theta=170^{\circ}$ on the benchmark pore geometry (Fig. 6). The pressure distribution in the invasion-percolation regime is spatially uniform within each fluid (Fig. 6d), with the two fluid pressures differing by the Laplace pressure. As the displacement front advances, the pressure in the invading fluid is modulated by the sequence of lowest capillary entry pressures, and fluctuates sharply (Fig. 6a) (Måløy et al. 1992; Furuberg et al. 1996). This intermittency is also reflected in the flow field: only a small fraction of the pore space is active at any given time (Fig. 6b), and the flow direction changes frequently (Fig. 6e). As a result, the emerging flow pattern lacks radial symmetry throughout the displacement, with invasion-time patches reflecting invasion avalanches (Fig. 6c).

\subsection{Cooperative Pore Filling $\left(D_{f}=1.93, w / a=15\right.$, and $\left.C a^{*}<1\right)$}

Cooperative pore filling is a capillary-dominated regime that produces compact displacement patterns. Although cooperative pore filling can take place in viscous flow regimes, they are most prominent in weak imbibition and can dominate the displacement pattern when viscous forces are small. During cooperative pore filling, the displacement front advances mainly through overlap and touch events (see §2), and the increased 

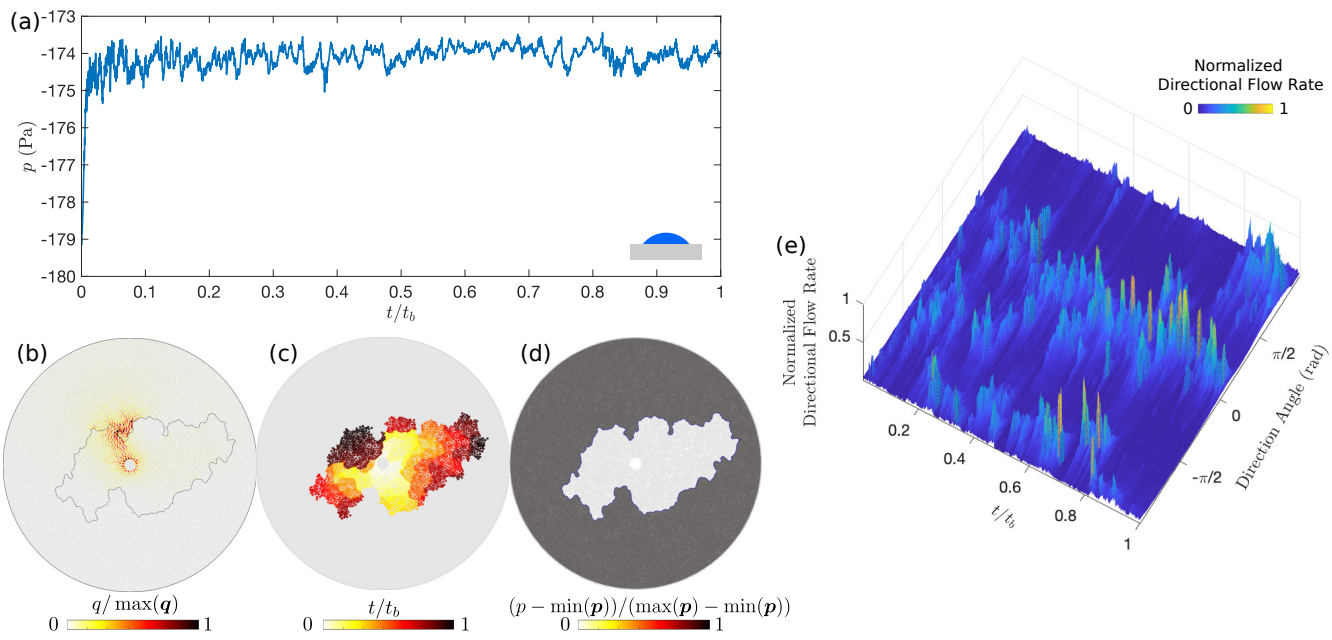

FiguRE 7. Cooperative pore filling in the benchmark geometry for $\mathrm{Ca}=10^{-7}, M=1$, and $\theta=46^{\circ}$; (a) the injection pressure is highly intermittent $\left(t_{b}\right.$ is the breakthrough time); (b) flowrates within the network are localized, and only a small fraction of them have appreciable flow; (c) pore-invasion times reveal pore-invasion clusters $(\max (\boldsymbol{q})$ is the largest local flowrate at given $t) ;(d)$ the pore-pressure distribution is uniform within each fluid phase $(\max (\boldsymbol{p})$ is the largest local pressure at given $t$ ); (e) the evolution of the directional flow rate shows a high degree of intermittency in the flow direction.

fraction of overlap events smooths the displacement front (Cieplak \& Robbins 1988, 1990; Holtzman \& Segre 2015; Primkulov et al. 2018). As a result, the displacement front sweeps the defending fluid completely, producing compact displacement patterns (Fig. 17).

Cooperative pore-filling simulations on the benchmark pore geometry at $\mathrm{Ca}=10^{-7}$, $M=1$, and $\theta=46^{\circ}$ (Fig. 7) show many similarities to invasion percolation ( $(3.3$ ). The pressure is uniform in each fluid phase (Fig. 7d), but exhibits sharp fluctuations in time (Fig. 7a). The flow field is highly intermittent (Fig. 7e), with only a small fraction of pores active at any given moment (Fig. 7b). This intermittency results in asymmetric and patch-like growth of the displacement pattern (Fig. 7c). Unlike invasion percolation, cooperative pore filling produces compact displacement patterns with no trapped patches of defending fluid. The difference stems from the nature of pore-scale invasion events: invasion percolation is dominated by burst events while cooperative pore filling is dominated by overlap and touch events (Cieplak \& Robbins 1988, 1990; Holtzman \& Segre 2015; Primkulov et al. 2018).

\subsection{Corner Flow $\left(D_{f}=1.54, w / a=0.8, C a^{*}<1\right)$}

In strong imbibition, the invading fluid no longer advances by filling the pores completely - instead, the invading fluid advances mainly through corner-flow events where it coats the corners at the intersection of posts with the top and bottom plates of the Hele-Shaw cell (Fig. 2c).

Fig. 8 explores corner flow through simulations at $\mathrm{Ca}=5 \cdot 10^{-7}, M=0.1$, and $\theta=4^{\circ}$ on the benchmark pore geometry. Corner flow shares many similarities with other capillary-dominated regimes. The spatial distribution of pressure is uniform within each fluid (Fig. 8d), while the injection pressure shows intermittency characteristic of capillary-dominated displacements (Fig. 8a). Only a small fraction of the pore space has 

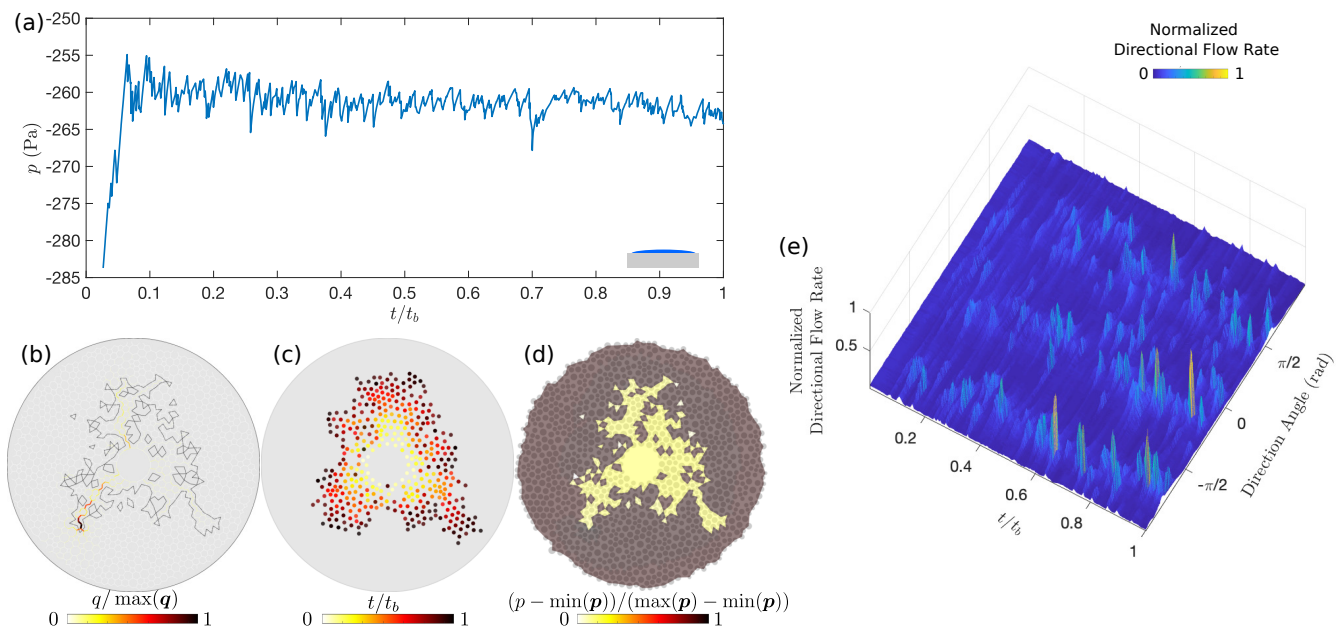

Figure 8. Corner flow in the benchmark geometry for $\mathrm{Ca}=5 \cdot 10^{-7}, M=0.1$, and $\theta=4^{\circ}$; (a) the injection pressure is highly intermittent ( $t_{b}$ is the breakthrough time); (b) flowrates within the network are localized, and only a small fraction of them have appreciable flow $(\max (\boldsymbol{q})$ is the largest local flowrate at given $t$ ); (c) pore-invasion times show radial asymmetry; (d) the pore-pressure distribution is uniform within each fluid phase $(\max (\boldsymbol{p})$ is the largest local pressure at given $t$ ); (e) the evolution of the directional flow rate shows a high degree of intermittency in the flow direction.

appreciable flow (Fig. 8b), and flow changes direction frequently (Fig. 8e). The resulting pattern grows asymmetrically throughout the displacement (Fig. 8c).

\section{Crossover from Viscous-Dominated to Capillary-Dominated Flow}

We examine the difference in the invasion dynamics between high and low Ca through the spatial and temporal distributions of pore-invasion events. In this section, we focus on unfavorable viscosity contrast displacement, $M=1 / 340$ (Zhao et al. 2016, 2019). The effective ratio of viscous to capillary forces is therefore $\mathrm{Ca} / M$, which we use in this section. Fig. 9a shows histograms of the Euclidean distance $\Delta s$ between consecutive poreinvasion events. The distribution of $\Delta s$ indicates that consecutive pore-invasion events are significantly more likely to take place near each other for low $\mathrm{Ca} / M$ than for high $\mathrm{Ca} / M$. Furthermore, the time $\Delta t_{\text {inv }}$ between consecutive pore-invasion events at $\mathrm{Ca} / M=10^{-7}$ shows that the median $\Delta t_{\text {inv }}$ increases as $\theta \rightarrow 46^{\circ}$ (Fig. 9b). As the wettability of the substrate changes from strong drainage to weak imbibition, the relative frequency of cooperative pore-filling events increases (Cieplak \& Robbins 1990, 1988; Primkulov et al. 2018). The increase in $\Delta t_{\text {inv }}$ is chiefly due to the increase in relative frequency of overlap events, which result in rapid invasion of several neighboring pores. This in turn leads to significant retraction of the invading fluid from all of the throats at the displacement front (see video S2 in supplementary materials). Thus, more time is needed to refill the pores at the displacement front, which results in the steady increase in $\Delta t_{\text {inv }}$ as $\theta$ decreases (Fig. 9b).

The velocity distribution within the porous medium is also strikingly different at low and high $\mathrm{Ca} / M$. We plot the temporal evolution of the directional flow rate for $\theta=46^{\circ}$ in Fig. 9(d-f). At $\mathrm{Ca} / M=10^{-3}$, the invading fluid forms high velocity flow channels that persist until breakthrough (Fig. 9d). The pressure gradients in the defending fluid dominate the dynamics, and the invading fluid flows through growing viscous fingers. The displacement front advances with strong radial symmetry (Fig. 9d and video S3), 

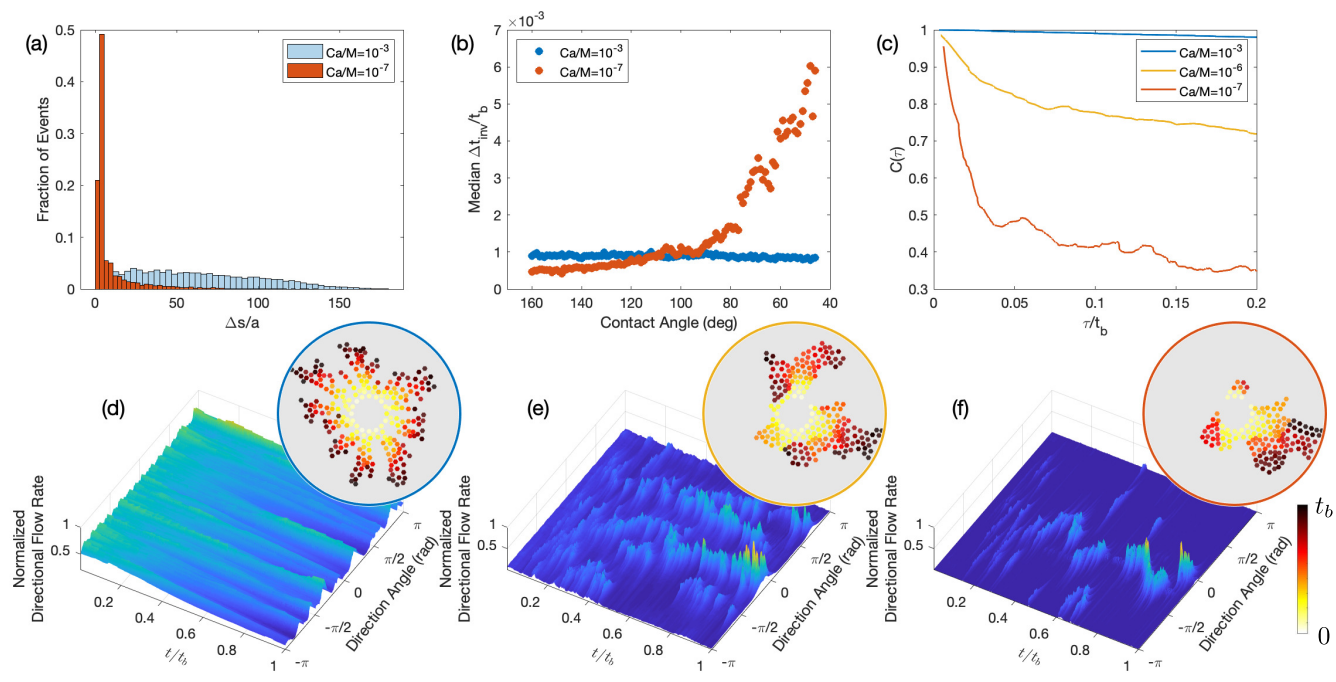

Figure 9. (a) Histogram of the distance $(\Delta s)$ between consecutive pore-invasion events. (b) Median time $\left(\Delta t_{\text {inv }}\right)$ between consecutive pore-invasion events as a function of $\theta$. (c) Spatiotemporal autocorrelation of the normalized directional flow rate fields for $\theta=46^{\circ}$. (d-f) Temporal evolution of the normalized directional-flow-rate fields for $\theta=46^{\circ}$ and (d) $\mathrm{Ca} / M=10^{-3}$, (e) $\mathrm{Ca} / M=10^{-6}$, (f) $\mathrm{Ca} / M=10^{-7}$. The plots are complemented with the pore invasion time diagrams (insets).

as observed experimentally (Måløy et al. 1985; Løvoll et al. 2004; Holtzman et al. 2012). As $\mathrm{Ca} / M$ decreases (Fig. 9e-f), the front velocity becomes increasingly intermittent. The pressure gradients within the fluids are negligible, and the pressure changes in the network are due almost exclusively to the Laplace pressure at the displacement front. Only portions of the displacement front are active at any given time (Holtzman et al. 2012; Ferer et al. 2004), and the front advances in asymmetric patches (Fig. 9e-f and video $\mathrm{S} 1$ ).

This transition from viscous-dominated to capillary-dominated flow can be quantified through the spatiotemporal autocorrelation of the normalized directional flow rate (Fig. 9c). The autocorrelation is calculated as $C(\alpha, \tau)=\frac{\langle q(\alpha, t) q(\alpha, t+\tau)\rangle}{\langle q(\alpha, t) q(\alpha, t)\rangle}$, where $\langle\cdot\rangle$ indicates the ensemble average over time, $\alpha$ is the direction, and $\tau$ is the time separation between the directional flow rate profiles. The average of $C(\alpha, \tau)$ over all $\alpha$ is shown in Fig. $9 \mathrm{c}$ for $\theta=46^{\circ}$. The flow field becomes increasingly uncorrelated at low $\mathrm{Ca} / M$, with a qualitative transition taking place below $\mathrm{Ca} / M=10^{-5}$.

\section{Extending Lenormand's Phase Diagram}

We extend Lenormand's diagram by simulating fluid-fluid displacement over a wide range of $\theta, \mathrm{Ca}, M$ on the benchmark pore geometry (7560 simulations in total). This thorough sweep of the parameter space is possible due to the relatively low computational cost of our model. For each simulation, we measure $D_{f}, w / a$, and $\mathrm{Ca}^{*}$ at the moment of breakthrough. We use these variables to delineate regions corresponding to the different principal flow regimes.

First, we use the fractal dimension $D_{f}$ to separate compact patterns from non-compact patterns. Compact patterns include stable displacement and cooperative pore filling, both of which have $D_{f}>1.92$ (maroon isosurface in Fig. 10d). A threshold based on $w / a$ provides similar results (not shown). 
16

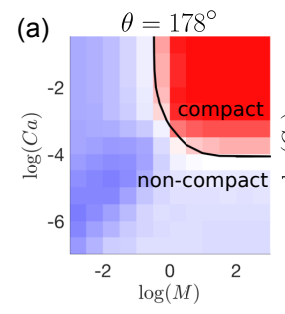

B.K. Primkulov et al.

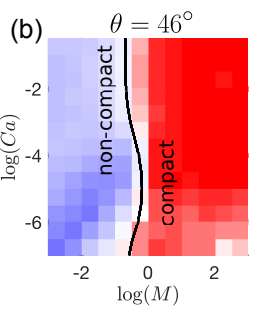

(d)

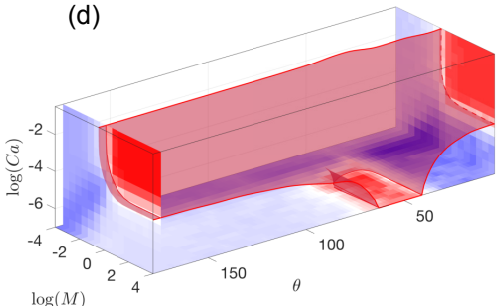

Figure 10. Evolution of $D_{f}$ in $M-\mathrm{Ca}-\theta$ space. Slices of the simulation data in (a) drainage, (b) weak imbibition, and (c) strong imbibition. (d) The maroon isosurface corresponding to $D_{f}=1.92$ is used to draw the boundary between compact and non-compact displacement patterns. The black lines are the intersections of the isosurface with the cross-sections.

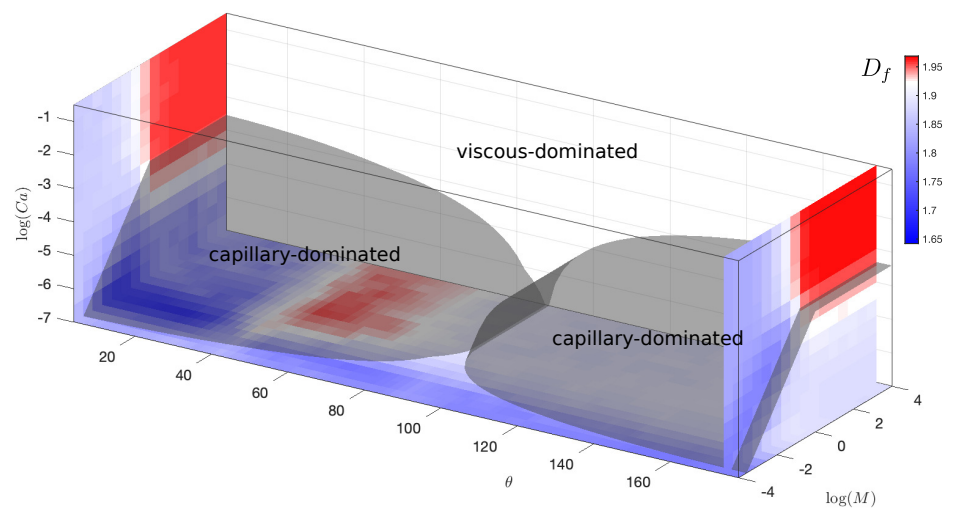

FiguRE 11 . Viscous-dominated and capillary-dominated regions of $M-\mathrm{Ca}-\theta$ space are separated by setting $\mathrm{Ca}^{*}=1$ in equation (3.1). This is depicted with a dark grey surface in this figure.

Next, we use $\mathrm{Ca}^{*}$ to separate viscous-dominated flow regions (stable displacement and viscous fingering) from capillary-dominated flow regions (cooperative pore filling, invasion percolation, and corner flow). The surface resulting from $\mathrm{Ca}^{*}=1$ in Eq. (3.1) is depicted in Figure 11 in dark grey: the space above this surface is viscous-dominated, the space below it is capillary-dominated. The crease on the $\mathrm{Ca}^{*}=1$ surface originates from vanishing out-of-plane contribution to Laplace pressure near $\theta=90^{\circ}$.

The combination of the maroon and gray isosurfaces from Figs. 10-11 is sufficient for delineating the principal flow regimes:

- invasion percolation is capillary dominated $\left(\mathrm{Ca}^{*}<1\right)$ and non-compact $\left(D_{f}<1.92\right)$;

- cooperative pore filling is capillary dominated $\left(\mathrm{Ca}^{*}<1\right)$ and compact $\left(D_{f}>1.92\right)$;

- corner flow is capillary dominated $\left(\mathrm{Ca}^{*}<1\right)$ and non-compact $\left(D_{f}<1.92\right)$;

- viscous fingering is viscous dominated $\left(\mathrm{Ca}^{*}>1\right)$ and non-compact $\left(D_{f}<1.92\right)$;

- stable displacement is viscous dominated $\left(\mathrm{Ca}^{*}>1\right)$ and compact $\left(D_{f}>1.92\right)$.

Although we use sharp boundaries to outline regions that belong to different flow regimes, the transitions from one regime to another are smooth, as is evident from the cross-section images in Figs. 10-11.

Our extension of Lenormand's diagram with added wettability axis is presented in Fig. 12. Our model faithfully reproduces the original diagram in drainage (cross-section $\theta=180^{\circ}$ in Fig. 12), but reveals a more complete picture of the fluid-fluid displacement in porous media by augmenting the phase diagram with a wettability $(\theta)$ axis.

To assess the influence of pore-scale disorder on the displacement pattern, we run simulations on a pore geometry in which we can precisely define, and tune, the degree 
of geometric variability among realizations. To do so, we generate a regular triangular lattice with $2.8 \mathrm{~mm}$ spacing between vertices and place posts on its vertices. The radii of the posts are drawn from a uniform distribution $\left(r_{0}-\xi r_{v}, r_{0}+\xi r_{v}\right)$, where $r_{0}=1100 \mu \mathrm{m}$ and $r_{v}=300 \mu \mathrm{m}$ are selected to match the mean post size of the benchmark geometry and $\xi \in[0,1]$ is the index of disorder. When $\xi=0$, the medium is ordered and anisotropic; when $\xi=1$, the medium is disordered and isotropic. As demonstrated in Appendix B, the values of $D_{f}$ and $w / a$ do not change significantly with the degree of disorder $\xi$. Therefore, although the data in Fig. 12 were collected from simulations on a single benchmark pore geometry, the results apply generally to porous media with varying degree of disorder. The capillary-dominated region of the phase diagram $\left(\mathrm{Ca}^{*}<1\right)$ is divided into invasion percolation, cooperative pore filling, and corner flow. The boundary between compact and non-compact flow in the capillary-dominated region of Fig. 12 changes significantly with Ca: the upper and lower bounds (in $\theta$ ) of the cooperative pore filling region move apart as $\mathrm{Ca}$ approaches the grey surface. When $M>1$, viscous forces stabilize the displacement front and aid cooperative pore filling events in making the patterns more compact ( $\mathrm{Hu}$ et al. 2018).

The shape of the extended Lenormand diagram can be inferred outside the $M-\mathrm{Ca}-\theta$ parameter space probed with the "moving-capacitor" model in Fig. 12. In particular, the cooperative pore filling region extends further into the $M<1$ region as Ca decreases. This is evident from the quasi-static limit of the model, where cooperative pore filling boundaries are independent of $M$.

The extended Lenormand diagram in Fig. 12 is generated for a single pore geometry. While the overall shape of the diagram is expected to hold across different micromodels with a wide range of pore-scale disorder, spacing between the posts, and gap thickness $h$, the boundaries between the principal flow regimes are likely to shift depending on the pore structure. For example, increasing the spacing between the post centers would bring the onset of cooperative pore filling to higher $\theta$ (Primkulov et al. 2018). Larger spacing between the posts would also make corner flow less dominant in strong imbibition, as higher critical pressures would be needed to coat post corners. Therefore, compact displacement would occupy a greater proportion of the overall space in Fig. 12. The degree of disorder is also known to roughen the displacement front and shift the boundary between invasion percolation and viscous fingering (Holtzman \& Juanes 2010; Holtzman 2016; $\mathrm{Hu}$ et al. 2019). Given that the pore geometry used in Figure 12 is similar to one with $\xi=0.99$ in Appendix B, a pore space with smaller degree of disorder would make compact displacement more favorable, which in turn would enlarge the compact displacement region in Fig. 12 (stable displacement and cooperative pore filling).

One should not think of the boundaries between the principal flow regimes in Fig. 12 as sharp, because transitions from one regime to another are gradual. Regions of the $M-$ $\theta-\mathrm{Ca}$ space near the maroon and grey boundaries correspond to crossover zones between principal flow regimes.

We summarize the findings from our comprehensive study with a schematic (Fig. 13). The classic phase diagram of Lenormand was developed for strong drainage (Fig. 13a), where displacement patterns advance through either viscous fingering, stable displacement, or invasion percolation. This diagram undergoes a qualitative change when the system moves to weak imbibition (Fig. 13b), in which viscous fingers become significantly wider and invasion percolation is replaced by cooperative pore filling. Therefore, the majority of the $M-\mathrm{Ca}$ space leads to compact displacement patterns. Strong imbibition has only been sparsely studied (Zhao et al. 2016; Odier et al. 2017; Primkulov et al. 2018), but enough is known to outline the main modes of displacement (Fig. 13c). The displacement patterns advance through corner flow at low $\mathrm{Ca}$, where the injected fluid 


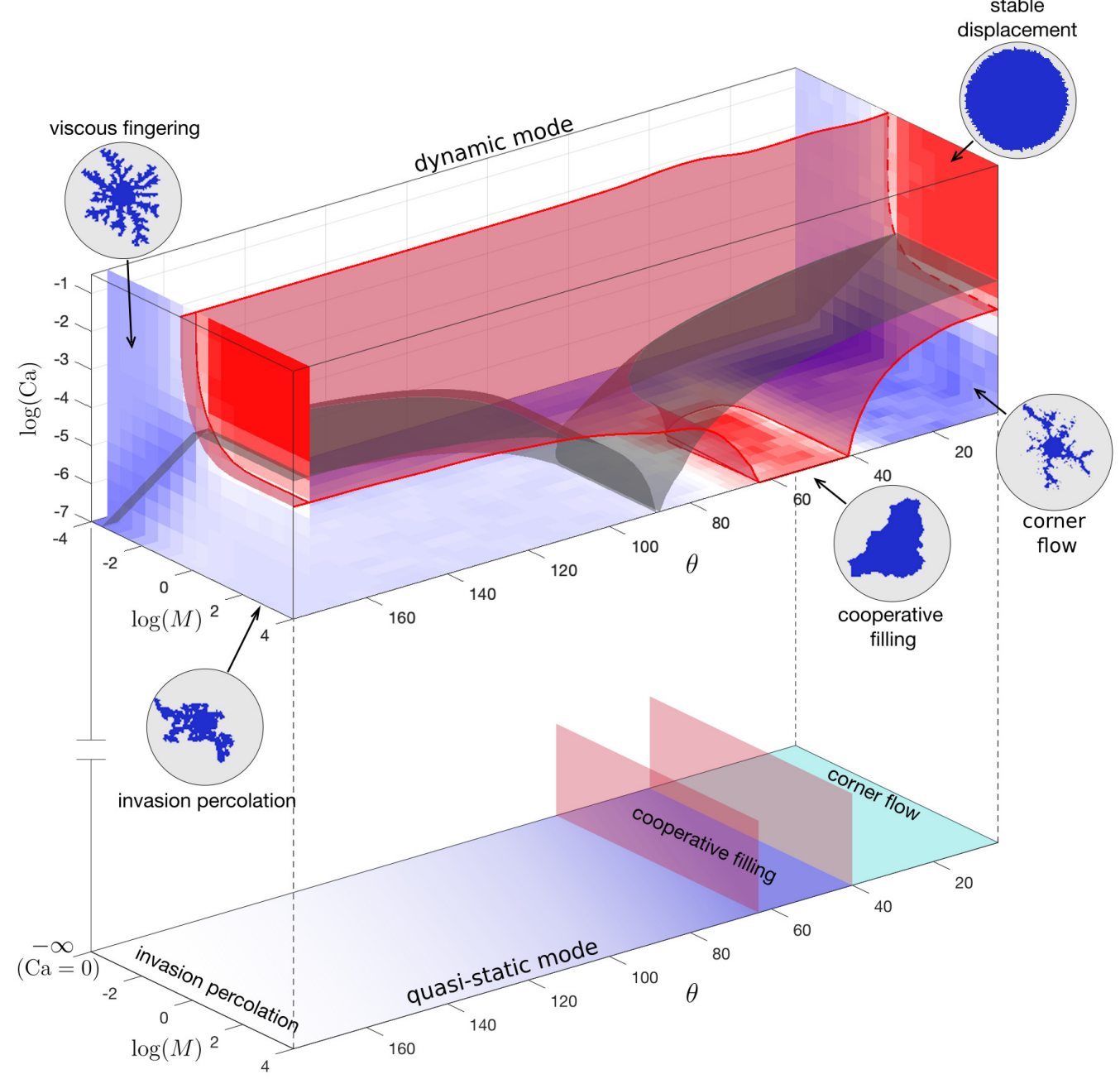

Figure 12. Extended Lenormand diagram constructed using $\mathrm{Ca}^{*}$ and $D_{f}$ phase boundaries from Figs. $10-11$ to separate the five principal flow regimes within the $M-\mathrm{Ca}-\theta$ parameter space: viscous fingering, stable displacement, invasion percolation, and cooperative pore filling. Results from the "moving-capacitor" model are complemented with results from the quasi-static model that allows inferring the extent of cooperative pore filling in the limit $\mathrm{Ca} \rightarrow 0$.

occupies only a fraction of the pore space (denoted by darker shades in Fig. 13c). This mode of displacement has been explored experimentally by Zhao et al. (2016) and Odier et al. (2017), and numerically in the quasi-static limit (Primkulov et al. 2018). The invasion pattern advances through thin films on the solid surface for high Ca and $M<1$ (Levaché \& Bartolo 2014), while maintaining the viscous fingering morphology (Zhao et al. 2016).

The simulations in Fig. 12 reproduce many experimental observations. First, as $\theta$ changes from $180^{\circ}$ to $46^{\circ}$, displacement patterns change from invasion percolation to cooperative pore filling (Trojer et al. 2015; Zhao et al. 2016), and finger width increases in the viscous-fingering region of the diagram (Stokes et al. 1986; Trojer et al. 2015; Zhao et al. 2016). Second, the injection pressure fluctuates sharply in capillary-dominated regimes (Måløy et al. 1992; Furuberg et al. 1996), but instead varies monotonically 

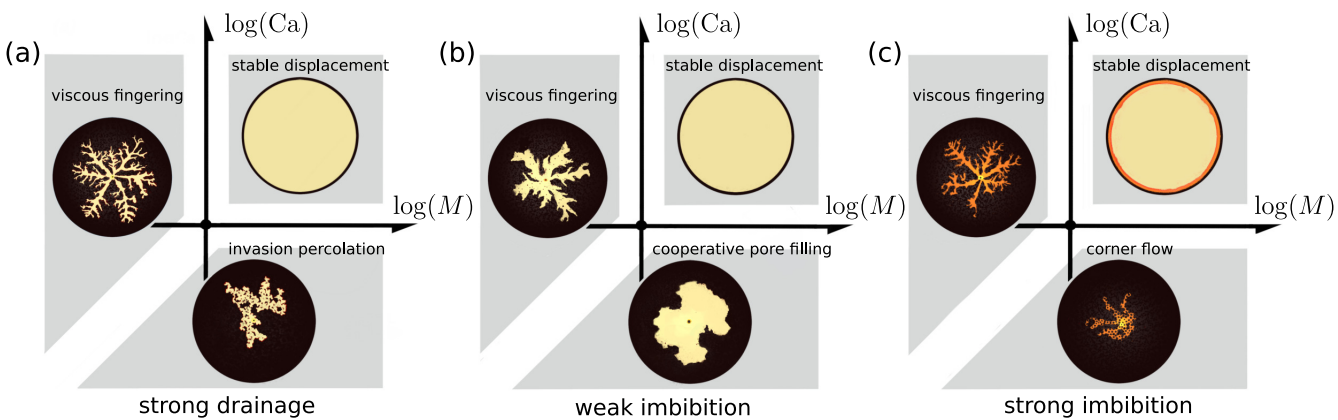

FiguRe 13. A sketch of Lenormand's phase diagram in (a) strong drainage, (b) weak imbibition and (c) strong imbibition. The darker shades in strong imbibition represent partial pore-scale displacement [art credit: Kamilla Omarova].

with time in viscous-dominated regimes. Third, the model naturally reproduces the intermittent flow that is modulated by pore disorder in capillary-dominated flow. Finally, the model reproduces the interplay between imposed ordered post lattice and the flow morphology: snow flake patterns in viscous fingering (Chen \& Wilkinson 1985; Chen 1987), regular crystal-growth morphology in cooperative pore filling regime (Lenormand 1990), perfect circles in stable displacement, and disordered morphology in invasion percolation (Lenormand \& Zarcone 1985; Wilkinson \& Willemsen 1983; Måløy et al. 1992).

While our "moving-capacitor" model is successful in reproducing the dynamics of the principal flow regimes (Fig. 13), it assumes piston-like displacement for burst, touch, and overlap events. As a result, the model overestimates the invading fluid saturation at high Ca, as pointed out by Zhao et al. (2019). Strong drainage and high Ca features residual films of defending fluid (Bretherton 1961; Zhao et al. 2016). In strong imbibition, invading fluid films dominate the displacement patterns in viscous fingering and corner flow regimes (Levaché \& Bartolo 2014; Zhao et al. 2016; Odier et al. 2017). These regimes are captured more naturally through pore-scale 3D continuum models (Zhao et al. 2019), which are unfortunately still prohibitively expensive for populating significant portions of the $M-\mathrm{Ca}-\theta$ parameter space in Lenormand's diagram (Fig. 12).

\section{Conclusion}

We have presented the results of a "moving-capacitor" dynamic pore-network model that is able to reproduce the full $M-$ Ca space of Lenormand's phase diagram and extend it with a third dimension $\theta$, thus accounting for the system's wettability. The model captures the pressure and flow within the porous medium, and our analysis of the model results shows the contrast in pore-scale dynamics between viscous-dominated and capillary-dominated flow through pore-invasion-event statistics and autocorrelation of the velocity field. The "moving-capacitor" model provides a single framework that captures the dynamics of fluid-fluid displacement in micromodels across an unprecedented span of $M-\mathrm{Ca}-\theta$ parameters. The model cannot be directly applied to generic porous materials with complex shapes or hierarchical geometries. However, in the spirit of Lenormand et al. (1988) and Cieplak \& Robbins (1988), here we studied a simpler pore geometry in order to learn something general about two-phase displacement in more complex porous media. We use our model to build the first three-dimensional version of Lenormand's phase diagram with wettability as the third axis, whose general shape we expect to hold for more complex three-dimensional porous materials. We demonstrate that cooperative 
pore filling can occupy a significant portion of $M-\mathrm{Ca}-\theta$ space, and that two metricsthe classical fractal dimension and modified capillary number $\mathrm{Ca}^{*}$ - are sufficient for delineating the five principal displacement regimes. One can use the diagram to design efficient fluid-fluid displacement in disordered porous media. Furthermore, the "movingcapacitor" model used in this work enables modeling multiphase flow in deformable granular media (movable posts) (Jain \& Juanes 2009; Sandnes et al. 2011; Lee et al. 2020), while accounting for the wettability effects, when combined with discrete element method (DEM) models (Meng et al. 2020).

\section{Acknowledgement}

Authors would like to thank Antoine Jacquey, Ehsan Haghighat, Josimar Alves da Silva Junior, Wei Li, Jun Li, and Peter Mora for insightful discussions and help with computation during project. This work was funded by the KFUPM-MIT collaborative agreement "Multiscale Reservoir Science". RJ acknowledges funding from the US Department of Energy (grant DE-SC0018357). Declaration of interests. The authors declare no conflict of interest.

\section{Appendix A. Fitting parameter for corner flow}

Ideal system. We first highlight how changes in $h$ - the height of posts within our micromodel-impact the onset of corner flow. This has been explored in earlier work (Primkulov et al. 2018), but we include it here for completeness. We examine the transition to corner flow in the quasi-static limit, where we set the outer radius of the micromodel to $15 \mathrm{~cm}$.

The out-of-plane contribution to Laplace pressure for burst, touch, and overlap events is a function of $h$ and reads as $-\frac{\gamma \cos \theta}{h / 2}$. Therefore, the total Laplace pressure of burst, touch, and overlap decreases with decreasing $h$. In contrast, the critical Laplace pressure of corner-flow event is independent of $h$ (Primkulov et al. 2018). In capillary-dominated displacement, events with lowest critical Laplace pressure take precedence. Therefore, the onset of corner flow depends on $h$. The impact of $h$ on the onset of corner flow at $\mathrm{Ca}=0$ is summarized in Figure 14. When the posts are infinitely tall $(h \rightarrow \infty)$, the mode of fluid-fluid displacement changes smoothly from invasion percolation to cooperative pore filling and then sharply to corner flow as wettability conditions change from drainage to weak and then strong imbibition. For $h \rightarrow \infty, \theta=39^{\circ}$ marks the onset of corner flow. Decreasing the value of $h$ moves the onset of corner flow towards lower $\theta$, until corner flow disappears altogether. Corner flow does not take place when $h=100 \mu \mathrm{m}$ in our micromodel.

Alternatively, one can shift the onset of corner flow by changing the spacing between the posts: narrower spacing would trigger corner flow at higher $\theta$. The Laplace pressure of a corner meniscus is a monotonically increasing function of its size: it increases from $-\infty$ to $\Delta p_{\text {crit }}$ as the meniscus volume increases from zero to its critical volume (Fig. $3 \mathrm{~d}$ ). Therefore, smaller spacing between the posts lowers critical Laplace pressures for cornerflow events and shifts the onset of corner flow to higher $\theta$. The changes in the spacing between the posts would also shift the transition from invasion percolation to cooperative filling (Primkulov et al. 2018), where wider spacing extends the cooperative pore filling regime to higher $\theta$.

Real system. We now compare the model outcomes to experimental data from Zhao et al. (2016). The major difference between the model and experiments is in the onset of corner flow: corner flow is the primary mode of capillary-dominated displacement in 


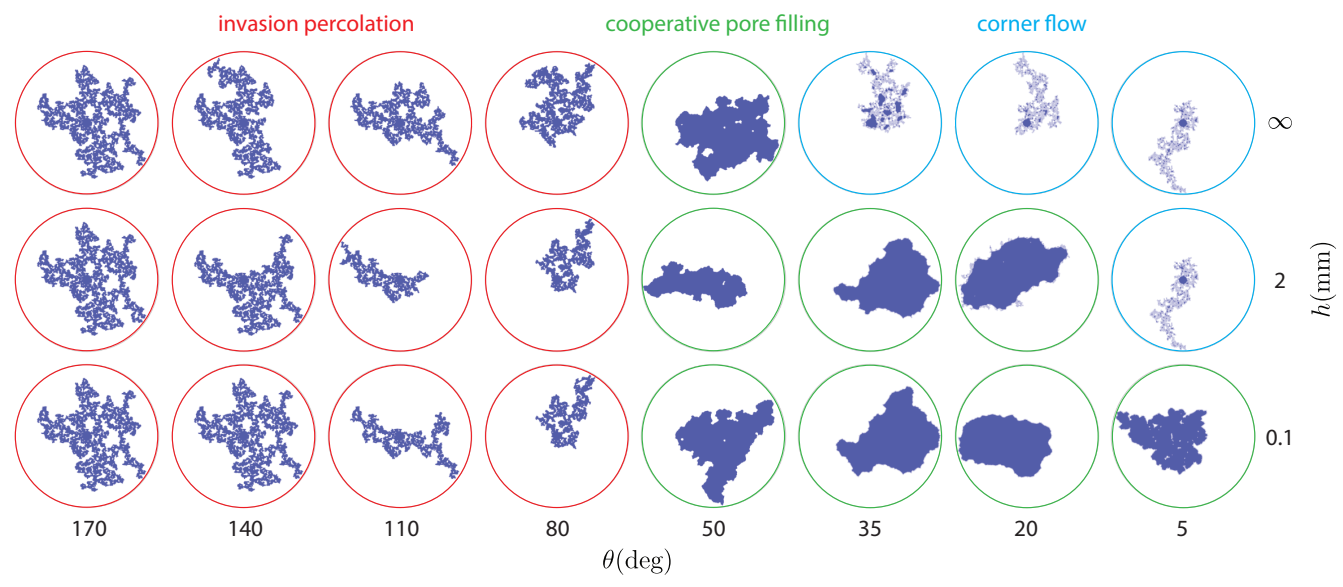

FiguRE 14. Transition to corner-flow regime as a function of post height $h$. Decreasing $h$ narrows the range of $\theta$ where corner flow dominates.

(a)

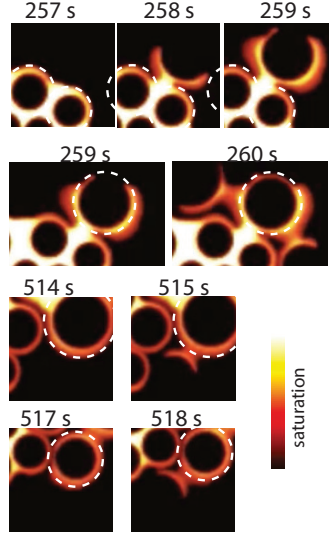

(b)

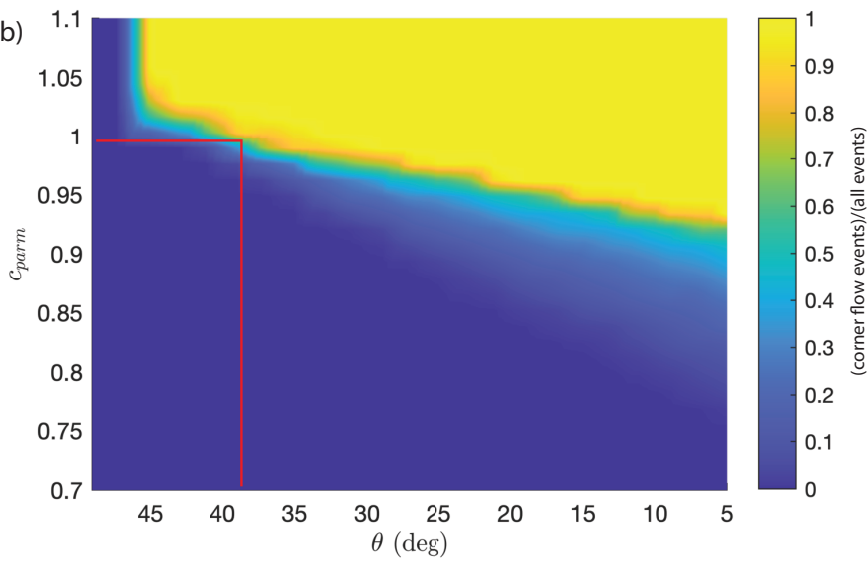

FiguRE 15. (a) Experimental image sequences of corner flow taken from Zhao et al. (2016) demonstrate instances where post-coating events take place before a circular portion of the corner meniscus swells to the extend of the nearby post; (b) changes in the fraction of corner flow events as a function of $\theta$ and the fitting parameter $c_{\text {parm }}$ are explored through the sweep of quasi-static simulations. The value of $c_{\text {parm }}$ used in this work and corresponding transition to corner flow are highlighted in red.

experiments with $h=100 \mu \mathrm{m}$ and $\theta=7^{\circ}$, while our model anticipates no corner flow for $h=100 \mu \mathrm{m}$ (Fig. 3d). In our model, corner flow is triggered when the horizontal radius of a corner meniscus reaches a neighboring uncoated post; this radius is marked $r_{n}$ in Fig. 3d. In the experiments, in contrast, neighboring posts are frequently coated well before the corner meniscus swells to the radius $r_{n}$ (Fig. 3d). Experiments suggest that more complex dynamics at the scale of the contact line can trigger the transition to corner flow.

While our model is strictly applicable for micromodels with ideal surfaces, the model can be tuned to match the experimentally observed onset of corner flow at $h=100 \mu \mathrm{m}$ by introducing a fitting parameter. Motivated by the observations in Fig. 15a, we can either trigger corner flow before the horizontal radius of a corner meniscus reaches $r_{n}$ or lower the critical Laplace pressure of corner flow events by out-of-plane curvature multiplied by 
coefficient $c_{\text {parm }}$. We chose the latter approach in this study. Setting $c_{\text {parm }}>0$ triggers earlier coating of the nearest posts through corner flow. We explore the sensitivity of our model to $c_{\text {parm }}$ in Fig. $15 \mathrm{~b}$ by reporting the fraction of corner-flow events as a function of $\theta$ and $c_{\text {parm }}$. We set $c_{\text {parm }}=1$ for the remainder of the discussion, which corresponds to a transition from cooperative pore filling to corner flow at $\theta=39^{\circ}$, in agreement with known experimental data (Zhao et al. 2016), where the transition from cooperative pore filling to corner flow takes place somewhere between $7^{\circ}$ and $60^{\circ}$.

The physical mechanisms behind the earlier onset of corner flow are not yet known. We speculate that since UV-treated NOA81 surfaces are highly hydrophilic (Levaché et al. 2012) and not ideally smooth, micron-scale water films may be present throughout the micromodel - between oil and the solid. This is in line with postulated film flow through micro-roughness by Vizika et al. (1994); Tzimas et al. (1997); Constantinides \& Payatakes (2000). However, since water saturation was tracked through concentration of the dye within the injected water phase in experiments of Zhao et al. (2016), detecting such films was not trivial. More detailed pore-scale studies are needed to fill this gap, where either water-sensitive dye is added to NOA81 or electric conductivity is utilized to sense pre-existing water films.

\section{Appendix B. Impact of Pore-Scale Disorder on Displacement Patterns}

Displacement patterns in each principal flow regime outlined in $\S 3$ interact with porescale disorder. We document this dependence briefly below.

Stable displacement. When $\mathrm{Ca}$ is sufficiently high and $M \gg 1$, the displacement pattern becomes insensitive to both wettability (given $\theta>45^{\circ}$ ) and disorder. The pattern is insensitive to wettability because viscosity dominates capillarity at high $\mathrm{Ca}$, and the pattern is insensitive to disorder because viscosity stabilizes the small perturbations from disorder.

Viscous fingering. In a circular Hele-Shaw cell without obstacles, the most unstable wavelength $\lambda$ of the instability follows (Saffman \& Taylor 1958)

$$
\frac{\lambda}{h}=\pi \sqrt{\frac{M}{\mathrm{Ca}(1-M)}},
$$

where $h$ is the spacing between the plates. In a radial Hele-Shaw cell, the number of viscous fingers with thickness $\lambda / 2$ increases with the radial distance from the center as the displacement evolves (Chen 1987, 1989).

Heterogeneity and anisotropy in the pore geometry can control the number of viscous fingers. In general, the degree of rotational symmetry of viscous fingers in ordered anisotropic media can be controlled by changing the post pattern. For instance, setting a rectangular lattice pattern on one plate of a circular Hele-Shaw cell promotes four-fold symmetry in finger growth (Chen 1987). A similar pattern occurs when posts are arranged on a rectangular lattice (Chen \& Wilkinson 1985). The simulations in Fig. 16 reproduce the results of the seminal work of Chen \& Wilkinson (1985), but on a triangular lattice. As $\xi$ increases from 0 to 1 , the invasion pattern moves away from the six-fold symmetry imposed by the lattice (Fig. 16a) (Holtzman 2016). The fractal dimension remains within the range $1.61<D_{f}<1.73$, consistent with experiments (Chen \& Wilkinson 1985; Måløy et al. 1985), while the finger width ranges from two to five pores $(2<w / a<5)$.

Whether the flow cell is ordered or disordered, wettability strongly influences the invasion patterns. Stokes et al. (1986) were the first to report that viscous fingers in 

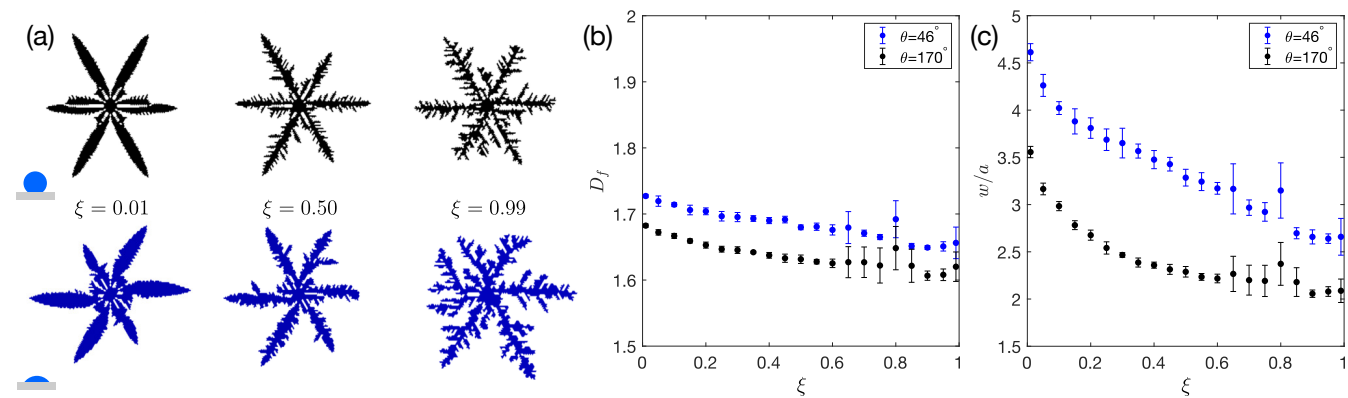

Figure 16. Viscous-fingering simulations $\left(\mathrm{Ca}=10^{-1}\right.$ and $\left.M=10^{-3}\right)$ conducted on a regular triangular lattice with varying degree of disorder $\xi$. (a) Black invasion patterns are in drainage $\left(\theta=170^{\circ}\right)$, blue patterns are in imbibition $\left(\theta=46^{\circ}\right)$. (b) Fractal dimension $D_{f}$ and (c) finger width $w / a$ are higher in imbibition across all degrees of disorder $\xi$. The error bars in (b-c) represent standard deviation of nine realizations.

imbibition are wider than in drainage. This observation has been confirmed in subsequent experimental studies (Trojer et al. 2015; Zhao et al. 2016; Lan et al. 2020). We observe the same trend for all degrees of disorder: both the finger width and the fractal dimension are consistently higher in imbibition than in drainage (Fig. 16b-c).

Invasion percolation. In this regime, the invading fluid preferentially enters pores with the lowest capillary entry pressures, one at a time. This process results in incomplete displacement of the defending fluid, which becomes trapped in clusters (Fig. 17, black). Both $D_{f}$ and $w / a$ of the resulting patterns remain nearly unaffected by the degree of disorder, with $1.61<D_{f}<1.79$ and $w / a \approx 3$ (Fig. 17b-c). Invasion percolation requires disorder in the throat sizes, but the actual degree of disorder does not matter when viscous forces are negligible $(\mathrm{Ca} \rightarrow 0)$. The lack of sensitivity of such invasion-percolation patterns to disorder is intuitive, as the pattern is ultimately determined only by the sequence in which pores are invaded. Therefore, a porous medium with small variations in throat size is equivalent to a porous medium with large variations in throat sizeonly the relative order of the throat sizes and their locations matter in shaping the invasion-percolation fronts. Therefore, unlike most fluid-fluid displacement regimes, it is very difficult to alter invasion-percolation patterns by imposing the order in the post lattice (see Fig. 17, black). This lack of sensitivity to disorder is likely responsible for the robustness and universality of the resulting patterns across different kinds of disordered media (Wilkinson \& Willemsen 1983; Cieplak et al. 1996; Sheppard et al. 1999).

Cooperative pore filling. Cooperative pore-filling events, which tend to smooth local concavities of the displacement front, allows patterns to be controlled by the post configuration. Slow injection of a wetting fluid into a porous medium with a regular triangular lattice results in a hexagonal invasion pattern (Fig. 17, blue). In fact, equivalents to our crystal-like patterns in imbibition and $\xi=0.01$ have been observed experimentally by Lenormand (1990). One can tune the displacement patterns to be squares, triangles (Lenormand 1990), and hexagons (Fig. 17, blue), via the lattice structure. Increasing $\xi$ makes the regular structure of the invading fluid become distorted.

Corner flow. Corner flow is remarkably similar to invasion percolation in how it interacts with disorder. While corner flow is sensitive to even mild disorder, it does not distinguish between different degrees of disorder, much like invasion percolation. Therefore corner flow is, in a sense, an analogue of invasion percolation for strong imbibition and may therefore possess universal features - producing robustly similar invasion pattern across different kinds of disordered media (Fig. 18). 

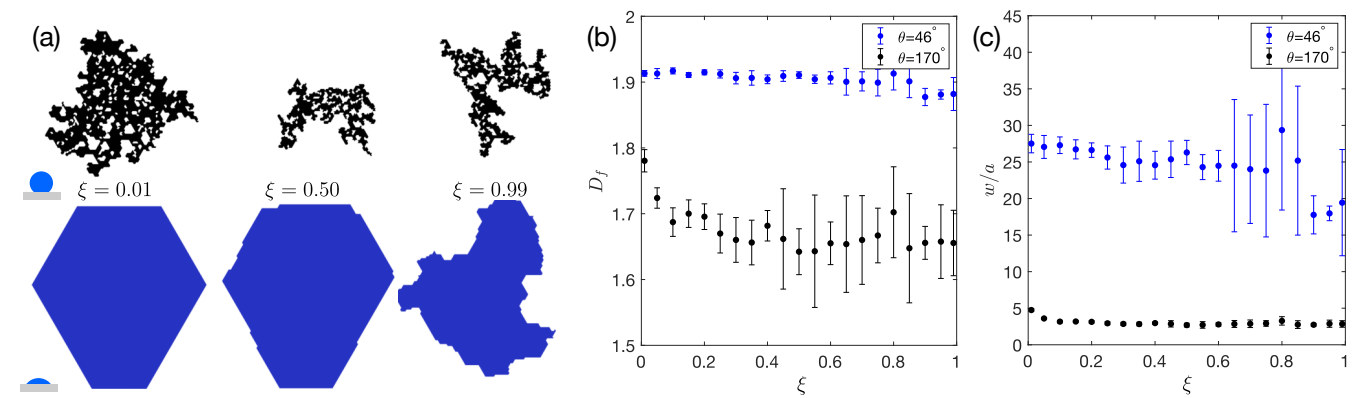

Figure 17. Capillary-dominated simulations $\left(\mathrm{Ca}=10^{-7}\right.$ and $\left.M=1\right)$ conducted on a regular triangular lattice with varying degree of disorder $\xi$. (a) Black invasion patterns are in drainage $\left(\theta=170^{\circ}\right)$ and correspond to invasion percolation, blue patterns are in imbibition $\left(\theta=46^{\circ}\right)$ and correspond to cooperative pore filling. (b) Fractal dimension $D_{f}$ and (c) finger width $w / a$ are higher in imbibition across all degrees of disorder $\xi$. The error bars in (b-c) represent standard the deviation of nine realizations.
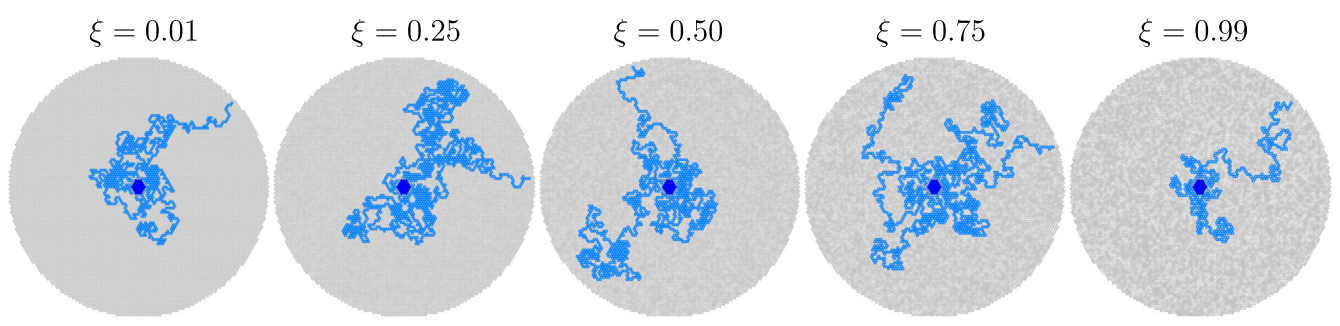

FiguRe 18. Quasi-static simulations in strong imbibition $\left(\theta=10^{\circ}\right)$ in a flow cell with a triangular post lattice and different degrees of disorder $\xi$. Dark blue regions represent fully invaded pores; light blue regions represent partially invaded pores with coated post corners.

\section{REFERENCES}

Aker, Eyvind, Måløy, Knut Jørgen, Hansen, Alex \& Batrouni, George 1998 A TwoDimensional Network Simulator for Two-Phase Flow in Porous Media. Transport in Porous Media 32 (2), 163-186.

Al-Gharbi, Mohammed S. \& Blunt, Martin J. 2005 Dynamic network modeling of twophase drainage in porous media. Physical Review E $\mathbf{7 1}$ (1), 016308.

Ben Amar, Martine 1991a Exact self-similar shapes in viscous fingering. Physical Review A $43(10), 5724-5727$.

Ben Amar, Martine $1991 b$ Viscous fingering in a wedge. Physical Review A 44 (6), 3673-3685.

Blunt, Martin J. 2001 Flow in porous media - pore-network models and multiphase flow. Current Opinion in Colloid 83 Interface Science 6 (3), 197-207.

Blunt, Martin J. \& Scher, Harvey 1995 Pore-level modeling of wetting. Physical Review E $\mathbf{5 2}(6), 6387-6403$.

Borgman, Oshri, Darwent, Thomas, Segre, Enrico, Goehring, Lucas \& Holtzman, RAN 2019 Immiscible fluid displacement in porous media with spatially correlated particle sizes. Advances in Water Resources 128, 158-167.

Bretherton, F. P. 1961 The motion of long bubbles in tubes. Journal of Fluid Mechanics 10, $166-188$.

Celia, Michael A., Reeves, Paul C. \& Ferrand, Lin A. 1995 Recent advances in pore scale models for multiphase flow in porous media. Reviews of Geophysics 33 (S2), 1049-1057.

Chandler, Richard, Koplik, Joel, Lerman, Kenneth \& Willemsen, Jorge F. 1982 Capillary displacement and percolation in porous media. Journal of Fluid Mechanics 119, 249-267.

Chen, J. D. 1987 Radial viscous fingering patterns in Hele-Shaw cells. Experiments in Fluids 5 (6), 363-371. 
Chen, Jing Den 1989 Growth of radial viscous fingers in a Hele-Shaw cell. Journal of Fluid Mechanics 201, 223-242.

Chen, Jing Den \& Wilkinson, David 1985 Pore-scale viscous fingering in porous media. Physical Review Letters 55 (18), 1892-1895.

Chuoke, R. L., van Meurs, P. \& van Der Poel, C. 1959 The Instability of Slow, Immiscible, Viscous Liquid-Liquid Displacements in Permeable Media .

Cieplak, Marek, Maritan, Amos \& Banavar, Jayanth R. 1996 Invasion percolation and Eden growth: Geometry and universality. Physical Review Letters 76 (20), 3754-3757.

Cieplak, Marek \& Robbins, Mark O. 1988 Dynamical Transition in Quasistatic Fluid Invasion in Porous Media. Physical Review Letters 60 (20), 2042-2045.

Cieplak, Marek \& Robbins, Mark O. 1990 Influence of contact angle on quasistatic fluid invasion of porous media. Physical Review B 41 (16), 11508-11521.

Constantinides, George N. \& Payatakes, Alkiviades C. 2000 Effects of precursor wetting films in immiscible displacement through porous media. Transport in Porous Media $\mathbf{3 8}$ (3), 291-317.

Conti, M. \& Marconi, U. M. B. 2010 Diffusion limited propagation of burning fronts. In WIT Transactions on Ecology and the Environment, , vol. 137, pp. 37-45. WIT Press.

Cox, R. G. 1986 The dynamics of the spreading of liquids on a solid surface. Part 1. Viscous flow. Journal of Fluid Mechanics 168, 169-194.

Datta, Sujit S., Ramakrishnan, T. S. \& Weitz, David A. 2014 Mobilization of a trapped non-wetting fluid from a three-dimensional porous medium. Physics of Fluids 26 (2), 22002 .

FATT, I. 1956 The Network Model of Porous Media. Petroleum Transactions, AIME 207, 144177.

Feder, Jens, Hinrichsen, Einar L., MÅløy, Knut Jørgen \& Jøssang, Torstein 1989 Geometrical crossover and self-similarity of DLA and viscous fingering clusters. Physica D: Nonlinear Phenomena 38 (1-3), 104-111.

Ferer, M., Ji, Chuang, Bromhal, Grant S., Cook, Joshua, Ahmadi, Goodarz \& Smith, DuAne H. 2004 Crossover from capillary fingering to viscous fingering for immiscible unstable flow: Experiment and modeling. Physical Review E 70 (1), 016303.

Furuberg, Liv, Måløy, Knut Jørgen \& Feder, Jens 1996 Intermittent behavior in slow drainage. Physical Review E 53 (1), 966-977.

Gjennestad, Magnus Aa., Vassvik, Morten, Kuelstrup, Signe \& Hansen, Alex 2018 Stable and Efficient Time Integration of a Dynamic Pore Network Model for Two-Phase Flow in Porous Media. Frontiers in Physics 6, 56.

Haines, William B. 1930 Studies in the physical properties of soil. V. The hysteresis effect in capillary properties, and the modes of moisture distribution associated therewith. The Journal of Agricultural Science 20 (1), 97-116.

Hill, S. 1952 Channeling in packed columns. Chemical Engineering Science 1 (6), 247-253.

Hinrichsen, E. L., MÅløy, K. J., Feder, J. \& Jøssang, T. 1989 Self-similarity and structure of DLA and viscous fingering clusters. Journal of Physics A: Mathematical and General 22 (7), 271-277.

Hoffman, Richard L 1975 A study of the advancing interface. I. Interface shape in liquid-gas systems. Journal of Colloid and Interface Science 50 (2), 228-241.

Holtzman, Ran 2016 Effects of Pore-Scale Disorder on Fluid Displacement in PartiallyWettable Porous Media. Scientific Reports 6 (1), 36221.

Holtzman, Ran \& JuAnes, Ruben 2010 Crossover from fingering to fracturing in deformable disordered media. Physical Review E 82 (4), 046305.

Holtzman, Ran \& Segre, Enrico 2015 Wettability Stabilizes Fluid Invasion into Porous Media via Nonlocal, Cooperative Pore Filling. Physical Review Letters 115 (16), 164501.

Holtzman, Ran, Szulczewski, Michael L. \& Juanes, Ruben 2012 Capillary Fracturing in Granular Media. Physical Review Letters 108 (26), 264504.

Homsy, G. M. 1987 Viscous Fingering in Porous Media. Annual Review of Fluid Mechanics 19 (1), 271-311.

Hu, Ran, Lan, Tian, Wei, Guan Ju \& Chen, Yi Feng 2019 Phase diagram of quasi-static immiscible displacement in disordered porous media. Journal of Fluid Mechanics 875, $448-475$. 
Hu, Ran, Wan, Jiamin, Yang, Zhibing, Chen, Yi-Feng \& Tokunaga, Tetsu 2018 Wettability and Flow Rate Impacts on Immiscible Displacement: A Theoretical Model. Geophysical Research Letters 45 (7), 3077-3086.

Jain, A. K. \& JuAnes, R. 2009 Preferential Mode of gas invasion in sediments: Grain-scale mechanistic model of coupled multiphase fluid flow and sediment mechanics. Journal of Geophysical Research 114 (B8), B08101.

Joekar-Niasar, V. \& Hassanizadeh, S. M. 2012 Analysis of Fundamentals of Two-Phase Flow in Porous Media Using Dynamic Pore-Network Models: A Review. Critical Reviews in Environmental Science and Technology 42 (18), 1895-1976.

Joekar-Niasar, V., Hassanizadeh, S. M. \& Dahle, H. K. 2010 Non-equilibrium effects in capillarity and interfacial area in two-phase flow: dynamic pore-network modelling. Journal of Fluid Mechanics 655, 38-71.

Jung, Michael, Brinkmann, Martin, Seemann, Ralf, Hiller, Thomas, Sanchez de La Lama, Marta \& Herminghaus, Stephan 2016 Wettability controls slow immiscible displacement through local interfacial instabilities. Physical Review Fluids 1 (7), 074202.

Kenkel, N. C. \& Walker, D. J. 1996 Fractals in the Biological Sciences. Coenoses 11, 77-100.

Lan, Tian, Hu, Ran, Yang, Zhibing, Wu, Dong-Sheng \& Chen, Yi-Feng 2020 Transitions of Fluid Invasion Patterns in Porous Media. Geophysical Research Letters 47 (20), e2020GL089682.

Landau, L. \& Levich, B. 1988 Dragging of a Liquid by a Moving Plate. In Dynamics of Curved Fronts, pp. 141-153. Elsevier.

Lee, Sungyon, Lee, Jeremy, Le Mestre, Robin, Xu, Feng \& MacMinn, Christopher W. 2020 Migration, trapping, and venting of gas in a soft granular material. Physical Review Fluids 5 (8), 084307.

Léger, Alain, Weber, Ludger \& Mortensen, Andreas 2015 Influence of the wetting angle on capillary forces in pressure infiltration. Acta Materialia 91, 57-69.

Lenormand, R 1990 Liquids in porous media. Journal of Physics: Condensed Matter 2 (S), SA79-SA88.

Lenormand, Roland, Touboul, Eric \& Zarcone, Cesar 1988 Numerical models and experiments on immiscible displacements in porous media. Journal of Fluid Mechanics 189, $165-187$.

Lenormand, Roland \& Zarcone, Cesar 1985 Invasion Percolation in an Etched Network: Measurement of a Fractal Dimension. Physical Review Letters 54 (20), 2226-2229.

Levaché, Bertrand, Azioune, Ammar, Bourrel, Maurice, Studer, Vincent \& BARTOLO, DENIS 2012 Engineering the surface properties of microfluidic stickers. Lab on a Chip 12 (17), 3028.

Levaché, Bertrand \& Bartolo, Denis 2014 Revisiting the Saffman-Taylor Experiment: Imbibition Patterns and Liquid-Entrainment Transitions. Physical Review Letters 113 (4), 044501.

Li, Shumang, Lowengrub, John S., Fontana, Jake \& Palffy-Muhoray, Peter 2009 Control of Viscous Fingering Patterns in a Radial Hele-Shaw Cell. Physical Review Letters 102 (17), 174501.

Løvoll, Grunde, Méheust, Yves, Toussaint, Renaud, Schmittbuhl, Jean \& Måløy, KNUT JøRGEN 2004 Growth activity during fingering in a porous Hele-Shaw cell. Physical Review E 70 (2), 12.

MÅløy, Knut Jørgen, Feder, Jens \& Jøssang, Torstein 1985 Viscous fingering fractals in porous media. Physical Review Letters 55 (24), 2688-2691.

Måløy, Knut Jørgen, Furuberg, Liv, Feder, Jens \& Jøssang, Torstein 1992 Dynamics of slow drainage in porous media. Physical Review Letters 68 (14), 2161-2164.

Meakin, P., Tolman, S. \& Blumen, A. 1989 Diffusion-Limited Aggregation. Proceedings of the Royal Society A: Mathematical, Physical and Engineering Sciences 423 (1864), 133148.

Meng, Yue, Primkulov, Bauyrzhan K., Yang, Zhibing, Kwok, Chung Yee \& Juanes, RUBEN 2020 Jamming transition and emergence of fracturing in wet granular media. Physical Review Research 2 (2), 022012.

MøLler, P. C.F. \& Bonn, D. 2007 The shear modulus of wet granular matter. EPL 80 (3), 38002 
Niemeyer, L., Pietronero, L. \& Wiesmann, H. J. 1984 Fractal Dimension of Dielectric Breakdown. Physical Review Letters 52 (12), 1033-1036.

Odier, Céleste, Levaché, Bertrand, Santanach-Carreras, Enric \& Bartolo, Denis 2017 Forced Imbibition in Porous Media: A Fourfold Scenario. Physical Review Letters 119 (20), 208005.

Øren, P. E., Bakke, Stig \& Arntzen, O. J. 1998 Extending Predictive Capabilities to Network Models. SPE Journal 3 (4), 324-336.

Park, C. W. \& Homsy, G. M. 1984 Two-phase displacement in Hele Shaw cells: Theory. Journal of Fluid Mechanics 139, 291-308.

PAterson, Lincoln 1984 Diffusion-limited aggregation and two-fluid displacements in porous media. Physical Review Letters 52 (18), 1621-1624.

Patmonoaji, Anindityo, Muharrik, Mushlih, Hu, Yingxue, Zhang, Chunwei \& Suekane, Tetsuya 2020 Three-dimensional fingering structures in immiscible flow at the crossover from viscous to capillary fingering. International Journal of Multiphase Flow 122, 103147.

PatzeK, T. W. 2001 Verification of a complete pore network simulator of drainage and imbibition. SPE Journal 6 (2), 144-156.

Primkulov, Bauyrzhan K., Pahlavan, Amir A., Fu, Xiaojing, Zhao, Benzhong, MacMinn, Christopher W. \& Juanes, Ruben 2019 Signatures of fluid-fluid displacement in porous media: wettability, patterns and pressures. Journal of Fluid Mechanics 875, R4.

Primkulov, Bauyrzhan K., Talman, Stephen, Khaleghi, Keivan, Rangriz Shokri, Alireza, Chalaturnyk, Rick, Zhao, Benzhong, Macminn, Christopher W. \& JuAnes, Ruben 2018 Quasistatic fluid-fluid displacement in porous media: Invasionpercolation through a wetting transition. Physical Review Fluids 3 (10), 104001.

Richefeu, Vincent, El Youssoufi, Moulay Saïd \& Radjaï, Farhang 2006 Shear strength properties of wet granular materials. Physical Review E $\mathbf{7 3}$ (5), 051304.

Saffman, P. G. \& Taylor, G. 1958 The Penetration of a Fluid into a Porous Medium or Hele-Shaw Cell Containing a More Viscous Liquid. Proceedings of the Royal Society A: Mathematical, Physical and Engineering Sciences 245 (1242), 312-329.

Sandnes, B., Flekkøy, E.G., Knudsen, H.A., MÅløy, K.J. \& See, H. 2011 Patterns and flow in frictional fluid dynamics. Nature Communications 2 (1), 288.

Sheppard, Adrian P., Knackstedt, Mark A., Pinczewski, W. V. \& Sahimi, Muhammad 1999 Invasion percolation: New algorithms and universality classes. Journal of Physics A: Mathematical and General 32 (49), 521-529.

Singh, Kamaljit, Scholl, Hagen, Brinkmann, Martin, Michiel, Marco Di, Scheel, Mario, Herminghaus, Stephan \& Seemann, Ralf 2017 The Role of Local Instabilities in Fluid Invasion into Permeable Media. Scientific Reports 7 (1), 444.

Stokes, J. P., Weitz, D. A., Gollub, J. P., Dougherty, A., Robbins, M. O., Chaikin, P. M. \& Lindsay, H. M. 1986 Interfacial Stability of Immiscible Displacement in a Porous Medium. Physical Review Letters 57 (14), 1718-1721.

Strang, Gilbert. 2007 Computational Science and Engineering. Wellesley-Cambridge Press.

Trojer, Mathias, Szulczewski, Michael L. \& Juanes, Ruben 2015 Stabilizing Fluid-Fluid Displacements in Porous Media Through Wettability Alteration. Physical Review Applied 3 (5), 054008.

Tzimas, G. C., Matsuura, T., Avraam, D. G., Van Der Brugghen, W., Constantinides, G. N. \& Payatakes, A. C. 1997 The combined effect of the viscosity ratio and the wettability during forced imbibition through nonplanar porous media. Journal of Colloid and Interface Science 189 (1), 27-36.

Valvatne, Per H. \& Blunt, Martin J. 2004 Predictive pore-scale modeling of two-phase flow in mixed wet media. Water Resources Research 40 (7), W07406.

Van Meurs, P. 1957 The Use of Transparent Three-Dimensional Models for Studying the Mechanism of Flow Processes in Oil Reservoirs. Transactions of the AIME 210 (01), 295-301.

Vizika, O., Avraam, D. G. \& Payatakes, A. C. 1994 On the role of the viscosity ratio during low-capillary-number forced imbibition in porous media. Journal of Colloid And Interface Science 165 (2), 386-401. 
Volnov, O. V. 1977 Hydrodynamics of wetting. Fluid Dynamics 11 (5), 714-721.

Wilkinson, D. \& Willemsen, J. F. 1983 Invasion percolation: a new form of percolation theory. Journal of Physics A: Mathematical and General 16 (14), 3365-3376.

Zhao, Benzhong, MacMinn, Christopher W. \& Juanes, Ruben 2016 Wettability control on multiphase flow in patterned microfluidics. Proceedings of the National Academy of Sciences 113 (37), 10251-10256.

Zhao, Benzhong, Macminn, Christopher W, Primkulov, Bauyrzhan K, Chen, Yu, Valocchi, Albert J, Zhao, Jianlin, Kang, Qinjun, Bruning, Kelsey, McClure, James E, Miller, Cass T, Fakhari, Abbas, Bolster, Diogo, Hiller, Thomas, Brinkmann, Martin, Cueto-Felgueroso, Luis, Cogswell, Daniel A, Verma, Rahul, Prodanović, Maša, Maes, Julien, Geiger, Sebastian, Vassvik, Morten, Hansen, Alex, Segre, Enrico, Holtzman, Ran, Yang, Zhibing, Yuan, Chao, Chareyre, Bruno \& Juanes, Ruben 2019 Comprehensive comparison of pore-scale models for multiphase flow in porous media. Proceedings of the National Academy of Sciences of the United States of America 116 (28), 13799-13806. 